\title{
Epidemiology of Sepsis-Associated Acute Kidney Injury in Beijing, China: A Descriptive Analysis
}

\author{
Haiman Wang (D) ${ }^{1, *}$ \\ Xiaojun Ji (iD ${ }^{1, *}$ \\ Amanda Ying Wang ${ }^{2-4}$ \\ Patrick Kevin $\mathrm{Wu}^{5}$ \\ Zhuang Liu (D) ' \\ Lei Dong' \\ Jingfeng Liu' \\ Meili Duan' \\ 'Department of Intensive Care Unit, \\ Beijing Friendship Hospital, Capital \\ Medical University, Beijing, I00050, \\ People's Republic of China; ${ }^{2}$ Division of \\ the Renal and Metabolic, George Institute \\ for Global Health, The University of New \\ South Wales, Sydney, New South Wales, \\ Australia; ${ }^{3}$ Concord Clinical School, the \\ University of Sydney, Sydney, Australia; \\ ${ }^{4}$ Department of Renal Medicine, \\ Concord Repatriation General Hospital, \\ Concord, Australia; ${ }^{5}$ Department of \\ Anaesthesiology, Queen Mary Hospital, \\ Hong Kong
}

*These authors contributed equally to this work
Correspondence: Meili Duan; Amanda Ying Wang

Email dmeili@ccmu.edu.cn;

awang@georgeinstitute.org.au
Background: Sepsis is the most common contributing factor towards development of acute kidney injury (AKI), which is strongly associated to poor prognostic outcomes. There are numerous epidemiological studies about sepsis-associated acute kidney injury (S-AKI), however current literature is limited with the majority of studies being conducted only in the intensive care unit (ICU) setting. The aim of this study was to assess the epidemiology of S-AKI in all hospitalized in-patients.

Methods: This was a retrospective population-based study using a large regional population database in Beijing city from January, 2005 to December, 2017. It included patients with S-AKI. Patients with pre-existing end-stage kidney disease (ESKD), previous history of kidney transplantation, or being pregnant were excluded. Patients' demographic characteristics, incidence, risk factors and outcomes of S-AKI were analyzed. The contrast between different time periods, different levels of hospitals, and types of the hospitals (traditional Chinese medicine hospitals (TCMHs) and western medicine hospitals (WMHs)) was also compared using Mann-Whitney $U$-test.

Results: A total of 19,579 patients were included. The overall incidence of S-AKI in all in-patients was $48.1 \%$. The significant risk factors by multivariate analysis for AKI included: age, male, being treated in a level-II hospital, pre-existing hypertension, chronic kidney disease (CKD), cirrhosis, atrial fibrillation (AF), ischemic heart disease (IHD), being admitted from emergency room, ICU admission, shock, pneumonia, intra-abdominal infection, bloodstream infection, respiratory insufficiency, acute liver injury, disseminated intravascular coagulation (DIC) and metabolic encephalopathy. The overall mortality rate in this cohort was 55\%. The multivariate analysis showed that the significant risk factors for mortality included: age, being treated in a level-II hospital and TCMHs, being admitted from emergency room, pre-existing comorbidities (CKD, malignancy, cirrhosis and AF), shock, pneumonia, intra-abdominal infection, bloodstream infection, central nervous system (CNS) infection and respiratory insufficiency.

Conclusion: AKI is a common complication in patients with sepsis, and its incidence increases over time, especially when ICU admission is required. Exploring interventional strategies to address modifiable risk factors will be important to reduce incidence and mortality of S-AKI.

Keywords: epidemiology, sepsis-associated acute kidney injury, traditional Chinese medicine hospitals, western medicine hospitals

\section{Background}

AKI is common in critically ill patients. Approximately one out of three patients in ICU develop AKI, and its incidence is increasing from $35.8 \%$ to $50.4 \%$. $^{1-5}$ 
Critically ill patients with AKI carries high mortality rates of up to $60 \%{ }^{6}$ Survivors following an episode of severe AKI in ICU have an increased risk of developing chronic kidney disease and end-stage kidney disease, cardiovascular disease and are associated with reduced quality of life. ${ }^{7,8}$

Sepsis remains the most common cause of AKI in the critically ill patients. ${ }^{7,9}$ There are numerous studies that have evaluated the epidemiology of sepsis-associated acute kidney injury (S-AKI). The largest study to date by Bagshaw et al, involving 57 Australian ICUs with $>120,000$ critically ill patients, reported a S-AKI incidence of only $11.7 \%{ }^{10}$ However, the incidence of S-AKI was reported higher in the Chinese literature, ranging from $45 \%$ to $51 \% .^{5,11,12}$ The difference in incidence could be attributed to differences in ethnic backgrounds and different AKI diagnostic criteria used in different studies. Furthermore, the existing studies on incidence of S-AKI in Chinese population were mainly derived from ICU data, which is not representative of the whole population. There is paucity of information on incidence and outcomes of S-AKI among different levels and types of healthcare system.

Therefore, the objective of this study was to assess the epidemiology of S-AKI in both ICU and non-ICU patients from all the level-II and tertiary hospitals in Beijing, China. The study also compared the differences between the level-II and tertiary level of hospitals as well as between TCMHs and WMHs on the incidence and outcomes of S-AKI, in order to provide greater insight into the burden of this condition in China, which is essential for the planning and assessment of interventional strategies to improve clinical outcomes of AKI patients.

\section{Methods}

\section{Data Source}

The data in this study was extracted from the Beijing Inpatient Registration System, ${ }^{13}$ which contains the diagnostic information of every inpatient in 16 districts and counties in Beijing, China.

According to the number of inpatient beds, it can be divided into three types of hospitals, level-I (20-99 beds), level-II (100-499 beds) and level-III (more than 500 beds). Because the number of level-one hospitals is small and the type of diseases that patients were admitted with are much different from those in the level-II and above hospitals, this study only included the data from the levelII and level-III hospitals for analyses.

\section{Study Population}

We conducted this retrospective study including hospitalized patients from 1 January, 2005 to 31 December, 2017 in Beijing, China. Patients with S-AKI were identified using the Primary International Classification of Diseases, 10th revision (ICD-10) codes.

The inclusion criteria consisted of: (1) adult patients (age $\geq 18$ years), (2) hospital stay longer than 24 hours in duration and (3) diagnosis consistent with sepsis (A41.9 based on ICD-10 classification). The exclusion criteria consisted of: (1) pre-existing end-stage kidney disease (ESKD), (2) patients who had received kidney transplantation or (3) pregnant patients. All methods in this study were performed in accordance with the relevant guidelines and regulations. This study was approved by the research ethics committee of Beijing Friendship Hospital (Reference Number: 2020-P2-089-01), and an informed consent was exempted by the same research ethics committee.

The reason for the waiver is based on the following statement from National Health Commission of the People's Republic of China, If the subject with identifiable information, whose material or data was used in the study, cannot be reached; and the research project does not involve any personal privacy or commercial interests, informed consent can be exempted by the research ethics committee.

Our study complies with the Declaration of Helsinki and all the patients' data included within our study were protected and maintained with confidentiality.

\section{Definitions of S-AKI}

Since the publication of the RIFLE consensus classification for AKI, modifications by the Acute Kidney Injury Network (AKIN), and Kidney Disease Improving Global Outcomes (KDIGO), these definitions have been commonly used in the majority of studies reporting on AKI. ${ }^{14-16}$

Our AKI definition followed the AKIN criteria from the 2005-2011 period and the KIDGO criteria from 2012 onwards. ${ }^{14,16}$ Given that the values of serum creatinine level and urine volume could not be obtained, the diagnosis of AKI was extrapolated directly from the database using the ICD-10 code. We used the A41.9 code as the diagnosis of sepsis and the N17.9 code as the diagnosis of AKI. 


\section{Statistical Analysis}

Patients' demographic characteristics were presented using proportions for categorical variables, mean (standard deviation) or median (interquartile range) as appropriate for continuous variables. Continuous variables were compared using the Student's $t$-test or Mann-Whitney $U$-test according to data distribution, while categorical variables were compared using the chi-square test.

Based on the literature study, variables available from the hospital discharge records and the results of univariate analysis, the following variables including age, age stratification, gender, hospital levels, time interval, comorbidities (including diabetes mellitus (DM), hypertension (HT), CKD stage 2 and stage 3, atrial fibrillation, ischemic heart disease, malignancy and cirrhosis), ${ }^{17-21}$ ICU admission, types of infection and types of organ function damage were selected in the logistic regression analysis to assess the risk factors of both the occurrence and mortality of S-AKI. ${ }^{4}$

Pre-specified subgroup analyses included level-II versus tertiary hospitals, TCMHs versus WMHs, and preKDIGO guideline era (2005-2011) and post-KDIGO guideline era (2012-2017).

Log rank tests were used to compare hospital survival rates between groups. A p value of $<0.05$ was considered statistically significant. Cox regression models were fitted to compare the mortality risk between groups adjusted for age, sex, medical insurance patterns, comorbidities, ICU admission, infection sites, shock and other organ dysfunction.

There were 220 cases $(0.54 \%)$ with missing hospitalization outcomes and 1 case $(0.0025 \%)$ with sepsis did not have expense variable. These cases were not included in the corresponding analysis.

All analyses were conducted with SAS 9.4 (SAS Institute Inc., Cary, North Carolina).

\section{Results}

A total of 40,720 septic patients were included in the final analysis, of which 19,579 met the criteria for S-AKI. The average age was 78 years old, with $62.7 \%$ were male patients. Majority of the patients $(90.1 \%)$ were admitted to tertiary hospitals generally from the emergency room $(60 \%)$.

Compared with septic patients without concurrent AKI, S-AKI patients were older, male predominant, and have more significant comorbidities (including HT, DM, CKD, cirrhosis, underlying malignancy, atrial fibrillation and ischemic heart disease).
Patients who experienced an episode of shock of any form (ie hypovolemic, cardiogenic or septic) were more susceptible to AKI. Majority of the patients (77.4\%) with S-AKI had pneumonia. Approximately, every 1 in 3 patients (34.3\%) with S-AKI required an ICU admission. Compared with septic patients without concurrent AKI, patients with S-AKI had longer hospital length of stays and were associated with significantly higher average daily costs $(\mathrm{P}<0.001)$ and overall costs $(\mathrm{P}<0.001)$. The demographic and clinical characteristics of these patients are presented in Table 1.

\section{Incidence and Risk Factors of S-AKI by Multivariate Analysis}

The incidence of S-AKI in all inpatients was $48.1 \%$, which was higher in ICU patients than non-ICU patients $(59.2 \%$ versus $31.6 \%, \mathrm{p}<0.001)$.

In multivariate logistic regression analysis, the following independent risk factors for S-AKI were identified (Table 2): age (odds ratio(OR) $=1.00,95 \%$ CI $(1.00$ 1.00), $\mathrm{P}<0.001)$, male ( $\mathrm{OR}=1.13,95 \%$ CI $(1.08-1.19)$, $\mathrm{P}<0.001)$, uninsured $(\mathrm{OR}=1.33$, 95\% CI $(1.47-1.22)$, $\mathrm{P}<0.001)$, being treated in a level-II hospital $(\mathrm{OR}=1.18$, 95\% CI (1.10-1.27), $\mathrm{P}<0.001)$, pre-existing hypertension $(\mathrm{OR}=1.20, \quad 95 \% \quad \mathrm{CI} \quad(1.14-1.26), \quad \mathrm{P}<0.001), \quad \mathrm{CKD}$ $(\mathrm{OR}=1.94, \quad 95 \% \quad \mathrm{CI} \quad(1.80-2.08), \quad \mathrm{P}<0.001)$, cirrhosis $(\mathrm{OR}=1.69,95 \%$ CI $(1.49-1.91), \mathrm{P}<0.001)$, atrial fibrillation $(\mathrm{OR}=1.28,95 \% \mathrm{CI}(1.20-1.36), \mathrm{P}<0.001)$, ischemic heart disease $(\mathrm{OR}=1.25,95 \% \mathrm{CI}(1.16-1.35), \mathrm{P}<0.001)$, being hospitalized from emergency department $(\mathrm{OR}=1.69$, 95\% CI (1.49-1.91), $\mathrm{P}<0.001)$, ICU admission $(\mathrm{OR}=1.2$, 95\% CI (1.14-1.26), $\mathrm{P}<0.001)$, shock (OR $=1.29,95 \% \mathrm{CI}$ (1.27-1.31), $\mathrm{P}<0.001)$, pneumonia $(\mathrm{OR}=1.33,95 \% \mathrm{CI}$ (1.18-1.49), $\quad \mathrm{P}<0.001), \quad$ intra-abdominal infection $(\mathrm{OR}=1.46,95 \%$ CI $(1.32-1.61), \mathrm{P}<0.001)$, bloodstream infection $(\mathrm{OR}=1.51,95 \%$ CI $(1.32-1.73), \quad \mathrm{P}<0.001)$, respiratory insufficiency $(\mathrm{OR}=1.93,95 \% \mathrm{CI}(1.84-2.03)$, $\mathrm{P}<0.001)$, acute liver injury ( $\mathrm{OR}=1.11,95 \%$ CI $(1.05-$ 1.17), $\mathrm{P}<0.001)$, DIC $(\mathrm{OR}=2.15,95 \%$ CI $(1.75-2.65)$, $\mathrm{P}<0.001)$ and metabolic encephalopathy $(\mathrm{OR}=1.93,95 \%$ CI (1.48-2.50), $\mathrm{P}<0.001)$.

\section{All-Cause Mortality and Risk Factors by Multivariate Analysis}

Overall all-cause mortality in S-AKI patients were 55\%. Majority of them (57.9\% of non-survivors) were admitted 
Table I Baseline Clinical Characteristics of Sepsis Patients with and without AKI

\begin{tabular}{|c|c|c|c|c|}
\hline Variables & & Sepsis with AKI $(n=19,579)$ & Sepsis without $\operatorname{AKI}(n=2 I, \mid 4 I)$ & $p$ value \\
\hline Age median [IQR] & & $78(64-84)$ & $75(59-83)$ & $<0.001$ \\
\hline Age stratification(years) & $\begin{array}{l}18-39 \\
40-59 \\
60-79 \\
\geq 80\end{array}$ & $\begin{array}{l}949(4.8) \\
2663(13.6) \\
7304(37.2) \\
8693(44.3)\end{array}$ & $\begin{array}{l}1703(8) \\
3633(17.2) \\
776 \mid(36.7) \\
8059(38.1)\end{array}$ & $<0.001$ \\
\hline Gender & $\begin{array}{l}\text { Female } \\
\text { Male }\end{array}$ & $\begin{array}{l}7294(37.3) \\
12,285(62.7)\end{array}$ & $\begin{array}{l}8272(39.1) \\
12,869(60.9)\end{array}$ & $<0.001$ \\
\hline Classification of insurance & $\begin{array}{l}\text { BMI } \\
\text { RCMTI } \\
\text { GMP } \\
\text { OPP } \\
\text { Others }\end{array}$ & $\begin{array}{l}10825(55.3) \\
1485(7.6) \\
3306(16.9) \\
1261(6.4) \\
2702(13.8)\end{array}$ & $\begin{array}{l}11,601(54.9) \\
1612(7.6) \\
3345(15.8) \\
1175(5.6) \\
3408(16.1)\end{array}$ & $<0.001$ \\
\hline Hospital level & $\begin{array}{l}\text { Tertiary hospital } \\
\text { Level-II hospital }\end{array}$ & $\begin{array}{l}17,519(89.5) \\
2060(10.5)\end{array}$ & $\begin{array}{l}19,151(90.6) \\
1990(9.4)\end{array}$ & $<0.001$ \\
\hline Hospital nature & $\begin{array}{l}\text { Western Medicine hospital } \\
\text { Hospital of TCM }\end{array}$ & $\begin{array}{l}18,006(92) \\
1573(8)\end{array}$ & $\begin{array}{l}19,4 \mid 2(91.8) \\
1729(8.2)\end{array}$ & 0.59 \\
\hline Time interval(year) & $\begin{array}{l}2005-2011 \\
2012-2017\end{array}$ & $\begin{array}{l}1468(7.5) \\
18,111(92.4)\end{array}$ & $\begin{array}{l}2228(10.5) \\
18,913(89.4)\end{array}$ & $<0.001$ \\
\hline Comorbidities(n) & $\begin{array}{l}\text { Diabetes } \\
\text { Hypertension } \\
\text { CKD stage } 2 \text { and } 3 \\
\text { Malignancy } \\
\text { Cirrhosis } \\
\text { Atrial fibrillation } \\
\text { Ischemic heart disease }\end{array}$ & $\begin{array}{l}7024(35.8) \\
10,227(52.2) \\
2944(15) \\
3750(19.1) \\
800(4.1) \\
3991(20.4) \\
2710(13.8)\end{array}$ & $\begin{array}{l}657 \mid(3|.|) \\
9573(45.2) \\
1655(7.8) \\
3832(\mid 8.1) \\
592(2.8) \\
2846(13.5) \\
1764(8.3)\end{array}$ & $\begin{array}{l}<0.001 \\
<0.001 \\
<0.001 \\
0.01 \\
<0.001 \\
<0.001 \\
<0.001\end{array}$ \\
\hline ICU & & $6725(34.3)$ & $4642(21.9)$ & $<0.001$ \\
\hline Types of shock & $\begin{array}{l}\text { No shock } \\
\text { Septic shock } \\
\text { Hypovolemic shock } \\
\text { Cardiac shock } \\
\text { Two or more kinds of shock }\end{array}$ & $\begin{array}{l}14,617(74.5) \\
3422(17.5) \\
988(5) \\
939(4.8) \\
345(1.8)\end{array}$ & $\begin{array}{l}19,233(90.9) \\
1073(5.1) \\
593(2.8) \\
357(1.7) \\
96(0.5)\end{array}$ & $\begin{array}{l}<0.001 \\
<0.001 \\
<0.001 \\
<0.001 \\
<0.001\end{array}$ \\
\hline Types of infection & $\begin{array}{l}\text { Pneumonia } \\
\text { Urinary infection } \\
\text { Intra-abdominal infection } \\
\text { CRBSI } \\
\text { CNS infection } \\
\text { Others }\end{array}$ & $\begin{array}{l}15,176(77.4) \\
1492(7.6) \\
1782(9.1) \\
657(3.4) \\
47(0.2) \\
3229(16.5)\end{array}$ & $\begin{array}{l}14,858(70.2) \\
1906(9) \\
1792(8.5) \\
472(2.2) \\
67(0.3) \\
4510(21.3)\end{array}$ & $\begin{array}{l}<0.001 \\
<0.001 \\
0.03 \\
<0.001 \\
0.14 \\
<0.001\end{array}$ \\
\hline Types of organ function damage & $\begin{array}{l}\text { Respiratory insufficiency } \\
\text { Acute liver injury } \\
\text { DIC } \\
\text { Metabolic encephalopathy }\end{array}$ & $\begin{array}{l}11,797(60.2) \\
4000(20.4) \\
680(3.5) \\
238(1.2)\end{array}$ & $\begin{array}{l}8032(38) \\
3518(16.6) \\
195(0.9) \\
124(0.6)\end{array}$ & $\begin{array}{l}<0.001 \\
<0.001 \\
<0.001 \\
<0.001\end{array}$ \\
\hline Expenses (Yuan) & $\begin{array}{l}\text { Total expense } \\
\text { Average daily expense } \\
\text { Western medicine expense } \\
\text { Chinese medicine expense }\end{array}$ & $\begin{array}{l}60,242.42(28,580.86-122,460.29) \\
5272.18(3 \mid 90.64-8583.87) \\
13,885.26(5645.02-30,424.3) \\
94.74(0-854.43)\end{array}$ & $\begin{array}{l}39,853.56(\mid 8,844.39-8 I, 420.7 I) \\
3224.33(\mid 862.96-5593.4) \\
9700.77(3298.62-22,050.47) \\
77.75(0-623.56)\end{array}$ & $\begin{array}{l}<0.001 \\
<0.001 \\
<0.001 \\
<0.001\end{array}$ \\
\hline
\end{tabular}

(Continued) 
Table I (Continued).

\begin{tabular}{|l|l|l|l|l|}
\hline Variables & & Sepsis with AKI (n=19,579) & Sepsis without AKI(n=2 I, I4I) & $\mathbf{P}$ value \\
\hline Outcome & Length of hospital stay (day) & $\begin{array}{l}13(6-23) \\
10,647(55)\end{array}$ & $\begin{array}{l}13(7-22) \\
6157(29.3)\end{array}$ & $<0.001$ \\
& Death(n) & $<0.00 I$ \\
\hline
\end{tabular}

Note: Values are expressed as the median (interquartile range and $\mathrm{N}(\%)$ ).

Abbreviations: BMI, basic medical insurance; RCMTI, rural cooperative medical treatment insurance; GMP, governmental medical payment; OPP, out-of-pocket payments; ER, emergency room; CRBSI, Catheter Related Blood Stream Infection; CNS, central nervous system; DIC, disseminated intravascular coagulation; TCM, traditional Chinese medicine.

Table 2 Risk Factors of Occurrence of S-AKI by Logistic Regression Analysis

\begin{tabular}{|c|c|c|c|c|}
\hline Variable & OR & $\begin{array}{l}\text { Lower } \\
95 \% \mathrm{Cl}\end{array}$ & $\begin{array}{l}\text { Higher } \\
95 \% \mathrm{Cl}\end{array}$ & $p$ value \\
\hline Age, years & 1.00 & 1.00 & 1.00 & $<0.001$ \\
\hline Male & 1.13 & 1.08 & 1.19 & $<0.001$ \\
\hline Uninsured & 1.33 & 1.47 & 1.22 & $<0.001$ \\
\hline Level-II hospital & 1.18 & 1.10 & 1.27 & $<0.001$ \\
\hline Treated in Western & 0.97 & 0.89 & 1.05 & 0.465 \\
\hline Medicine hospitals & & & & \\
\hline Diabetes & 1.03 & 0.98 & 1.08 & 0.263 \\
\hline Hypertension & 1.20 & 1.14 & 1.26 & $<0.001$ \\
\hline CKD stage 2 and 3 & 1.94 & 1.80 & 2.08 & $<0.001$ \\
\hline Malignancy & 1.06 & 1.00 & 1.12 & 0.058 \\
\hline Cirrhosis & 1.69 & 1.49 & 1.91 & $<0.001$ \\
\hline Atrial fibrillation & 1.28 & 1.2 & 1.36 & $<0.001$ \\
\hline Ischemic heart disease & 1.25 & 1.16 & 1.35 & $<0.001$ \\
\hline $\begin{array}{l}\text { Admission from } \\
\text { emergency department }\end{array}$ & 1.69 & 1.49 & 1.91 & $<0.001$ \\
\hline ICU admission & 1.20 & 1.14 & 1.26 & $<0.001$ \\
\hline Shock & 1.29 & 1.27 & 1.31 & $<0.001$ \\
\hline Pneumonia & 1.33 & 1.18 & 1.49 & $<0.001$ \\
\hline Urinary infection & 0.77 & 0.70 & 0.84 & $<0.001$ \\
\hline Intra-abdominal infection & 1.46 & 1.32 & 1.61 & $<0.001$ \\
\hline Bloodstream infection & 1.51 & 1.32 & 1.73 & $<0.001$ \\
\hline CNS infection & 1.19 & 0.79 & 1.79 & 0.403 \\
\hline Respiratory insufficiency & 1.93 & 1.84 & 2.03 & $<0.001$ \\
\hline Acute liver injury & I.II & 1.05 & 1.17 & $<0.001$ \\
\hline DIC & 2.15 & 1.75 & 2.65 & $<0.001$ \\
\hline Metabolic encephalopathy & 1.93 & 1.48 & 2.50 & $<0.001$ \\
\hline
\end{tabular}

Abbreviations: CKD, chronic kidney disease; CNS, central nervous system; DIC, disseminated intravascular coagulation.

to ICU. The mortality of patients admitted to ICU was lower than those who were not $(53.4 \%$ versus $54.8 \%$, $\mathrm{P}<0.001$ ).

Patients with pneumonia had significantly higher mortality rates than those without $(\mathrm{P}<0.001)$. Patients with multi-functional organ dysfunction syndrome was also associated with increased mortality, and had significantly higher overall costs $(\mathrm{p}=0.02)$ and average daily costs $(\mathrm{P}<$ 0.001) (Table 3).
A multivariate regression analysis revealed that risk factors for mortality included (Table 4): age (OR=1.02, 95\% CI (1.02$1.02), \mathrm{P}<0.001)$, being treated in a level-II hospital $(\mathrm{OR}=1.57$, 95\% CI (1.42-1.74), $\mathrm{P}<0.001)$, being hospitalized from emergency department $(\mathrm{OR}=1.14,95 \% \mathrm{CI}(1.07-1.22), \mathrm{P}<$ $0.001)$, pre-existing comorbidities $\mathrm{CKD}(\mathrm{OR}=1.24,95 \% \mathrm{CI}$ (1.14-1.36), $\mathrm{P}<0.001)$, malignancy $(\mathrm{OR}=2.03,95 \% \mathrm{CI}(1.86-$ $2.23), \mathrm{P}<0.001)$, cirrhosis $(\mathrm{OR}=1.75,95 \% \mathrm{CI}(1.45-2.11), \mathrm{P}<$ $0.001)$, atrial fibrillation ( $\mathrm{OR}=1.21,95 \% \mathrm{CI}(1.12-1.31), \mathrm{P}<$ $0.001)$, shock $(\mathrm{OR}=1.45,95 \% \mathrm{CI}(1.42-1.49), \mathrm{P}<0.001)$, pneumonia $(\mathrm{OR}=1.56,95 \% \mathrm{CI}(1.33-1.83), \mathrm{P}<0.001)$, intraabdominal infection ( $\mathrm{OR}=1.61,95 \% \mathrm{CI}(1.41-1.83), \mathrm{P}<$ $0.001)$, bloodstream infection $(\mathrm{OR}=1.34,95 \%$ CI $(1.12-$ 1.59), $\mathrm{P}<0.001)$, CNS infection ( $\mathrm{OR}=2.27,95 \% \mathrm{CI}(1.20$ $4.30), \mathrm{P}=0.012)$, and respiratory insufficiency $(\mathrm{OR}=1.87,95 \%$ CI (1.75-2.0), $\mathrm{P}<0.001)$.

We tested collinearity for relevant variables models and there was no collinearity among the included variables.

The development of AKI strongly influenced hospital survival rates in patients with sepsis $(45.0 \%$ versus $70.7 \%$ in patients with and without AKI, respectively; $\mathrm{p}<0.001$; Figure 1).

After adjusting for the confounding factors mentioned in the statistical analysis section, the results from the Cox proportional hazards model showed that septic patients with AKI had a significantly higher risk of death than those without (HR=1.47, 95\% CI $(1.42-1.53), \mathrm{P}<0.001)$.

\section{S-AKI Before and After the KDIGO Guideline Era (Table 5)}

The incidence of S-AKI post the KDIGO guideline era was higher than pre-KDIGO (48.9\% versus $39.7 \%$, P < 0.001 ), but the mortality rate was significantly lower after the KDIGO guideline era $(54.4 \%$ versus $63.9 \%$, p $<0.001$, Figure 2). Comparing with the patients before the KDIGO guideline era (period 2005-2011), patients after the KDIGO guideline era (2012-2017) were older $(\mathrm{p}<$ $0.001)$, but no gender difference was observed $(\mathrm{p}=0.69)$. 
Table 3 Baseline Clinical Characteristics of Survivors and Non-Survivors of S-AKI Patients

\begin{tabular}{|c|c|c|c|c|}
\hline Variables & & Survivors & Non-Survivors & $p$ value \\
\hline N (\%) & & $87 \mid 2(45.0)$ & $10,647(55.0)^{*}$ & \\
\hline Age median [IQR] & & $76(60-84)$ & $79(68-85)$ & $<0.001$ \\
\hline Age stratification(years) & $\begin{array}{l}18-39 \\
40-59 \\
60-79 \\
\geq 80\end{array}$ & $\begin{array}{l}575(6.6) \\
1487(17.1) \\
3212(36.9) \\
3438(39.5)\end{array}$ & $\begin{array}{l}358(3.4) \\
1134(10.7) \\
3983(37.4) \\
5172(48.6)\end{array}$ & $<0.001$ \\
\hline Gender & $\begin{array}{l}\text { Female } \\
\text { Male }\end{array}$ & $\begin{array}{l}3182(36.5) \\
5530(63.5)\end{array}$ & $\begin{array}{l}4029(37.8) \\
6618(62.2)\end{array}$ & 0.06 \\
\hline Classification of insurance & $\begin{array}{l}\text { BMI } \\
\text { RCMTI } \\
\text { GMP } \\
\text { OPP } \\
\text { Others }\end{array}$ & $\begin{array}{l}3929(45.1) \\
958(11) \\
1890(21.7) \\
622(7.1) \\
1313(15.1)\end{array}$ & $\begin{array}{l}6818(64) \\
483(4.5) \\
1415(13.3) \\
639(6) \\
1292(12.1)\end{array}$ & $<0.001$ \\
\hline Hospital level & $\begin{array}{l}\text { Tertiary hospitals } \\
\text { Second-class hospital }\end{array}$ & $\begin{array}{l}7953(91.3) \\
759(8.7)\end{array}$ & $\begin{array}{l}9370(88) \\
1277(12)\end{array}$ & $<0.001$ \\
\hline Hospital nature & $\begin{array}{l}\text { Western Medicine hospital } \\
\text { Hospital of TCM }\end{array}$ & $\begin{array}{l}8200(94.1) \\
512(5.9)\end{array}$ & $\begin{array}{l}9603(90.2) \\
1044(9.8)\end{array}$ & 0.59 \\
\hline Time interval(year) & $\begin{array}{l}2005-2011 \\
2012-2017\end{array}$ & $\begin{array}{l}450(5.2) \\
8262(94.8)\end{array}$ & $\begin{array}{l}798(7.5) \\
9849(92.5)\end{array}$ & $<0.001$ \\
\hline Comorbidities(n) & $\begin{array}{l}\text { Diabetes } \\
\text { Hypertension } \\
\text { CKD stage } 2 \text { and } 3 \\
\text { Malignancy } \\
\text { Cirrhosis } \\
\text { Atrial fibrillation } \\
\text { Ischemic heart disease }\end{array}$ & $\begin{array}{l}3475(39.9) \\
4785(54.9) \\
1134(13) \\
1288(14.8) \\
305(3.5) \\
2406(22.6) \\
1183(13.6)\end{array}$ & $\begin{array}{l}3513(33) \\
5394(50.7) \\
1804(16.9) \\
2430(22.8) \\
495(4.6) \\
1584(18.2) \\
1518(14.3)\end{array}$ & $\begin{array}{l}<0.001 \\
<0.001 \\
<0.001 \\
<0.001 \\
<0.001 \\
<0.001 \\
0.18\end{array}$ \\
\hline ICU & & $3136(36)$ & $3589(33.7)$ & $<0.001$ \\
\hline Types of shock & $\begin{array}{l}\text { No shock } \\
\text { Septic shock } \\
\text { Hypovolemic shock } \\
\text { Cardiac shock } \\
\text { Two or more kinds of shock }\end{array}$ & $\begin{array}{l}7179(82.4) \\
1032(11.8) \\
380(4.4) \\
190(2.2) \\
67(0.8)\end{array}$ & $\begin{array}{l}7400(69.5) \\
2178(20.5) \\
607(5.7) \\
745(7) \\
273(2.6)\end{array}$ & $\begin{array}{l}<0.001 \\
<0.001 \\
<0.001 \\
<0.001 \\
<0.001\end{array}$ \\
\hline Types of infection & $\begin{array}{l}\text { Pneumonia } \\
\text { Urinary infection } \\
\text { Intra-abdominal infection } \\
\text { CRBSI } \\
\text { CNS infection } \\
\text { Others }\end{array}$ & $\begin{array}{l}6508(74.7) \\
898(10.3) \\
794(9.1) \\
275(3.2) \\
20(0.2) \\
1568(18)\end{array}$ & $\begin{array}{l}8527(80.1) \\
576(5.4) \\
967(9.1) \\
377(3.5) \\
27(0.3) \\
1580(14.8)\end{array}$ & $\begin{array}{l}<0.001 \\
<0.001 \\
0.94 \\
0.14 \\
0.74 \\
<0.001\end{array}$ \\
\hline Types of organ function damage & $\begin{array}{l}\text { Respiratory insufficiency } \\
\text { Acute liver injury } \\
\text { DIC } \\
\text { Metabolic encephalopathy }\end{array}$ & $\begin{array}{l}457 \mid(52.5) \\
1780(20.4) \\
205(2.4) \\
I I 2(I .3)\end{array}$ & $\begin{array}{l}7135(67) \\
2199(20.7) \\
473(4.4) \\
126(1.2)\end{array}$ & $\begin{array}{l}<0.001 \\
0.7 \\
<0.001 \\
0.52\end{array}$ \\
\hline
\end{tabular}


Table 3 (Continued).

\begin{tabular}{|c|c|c|c|c|}
\hline Variables & & Survivors & Non-Survivors & $p$ value \\
\hline Expenses (Yuan) & $\begin{array}{l}\text { Total expense } \\
\text { Average daily expense } \\
\text { Western medicine expense } \\
\text { Chinese medicine expense }\end{array}$ & $\begin{array}{l}58,362.5(29,533.66-|| 4,864.4) \\
4673.4 \mid(268 \mid .64-7898.79) \\
\mid 3,422.67(5867.98-28,107.39) \\
\mid 42.69(0-1087.74)\end{array}$ & $\begin{array}{l}62,520.49(27,564.02-\mid 30,377.97) \\
5823.35(3670.6|-9| 87.57) \\
\mid 4,098.64(54 \mid 4.67-32,046.23) \\
68.98(0-693.93)\end{array}$ & $\begin{array}{l}0.02 \\
<0.001 \\
0.01 \\
<0.001\end{array}$ \\
\hline Outcome & Length of hospital stay (day) & I4(7-24) & $12(4-22)$ & $<0.001$ \\
\hline
\end{tabular}

Notes: *220 missing values because of lacking of outcome indicators. Values are expressed as the median (interquartile range and $\mathrm{N}(\%)$ ).

Abbreviations: BMI, basic medical insurance; RCMTI, rural cooperative medical treatment insurance; GMP, governmental medical payment; OPP, out-of-pocket payments; ER, emergency room; CRBSI, Catheter Related Blood Stream Infection; CNS, central nervous system; DIC, disseminated intravascular coagulation; TCM, traditional Chinese medicine.

Table 4 Risk Factors of Mortality of S-AKI by Logistic Regression Analysis

\begin{tabular}{|c|c|c|c|c|}
\hline Variable & OR & $\begin{array}{l}\text { Lower } \\
95 \% \mathrm{Cl}\end{array}$ & $\begin{array}{l}\text { Higher } \\
95 \% \mathrm{Cl}\end{array}$ & $p$ value \\
\hline Age, years & 1.02 & 1.02 & 1.02 & $<0.001$ \\
\hline Male gender & 0.96 & 0.90 & 1.02 & 0.207 \\
\hline Insured & 1.03 & 0.90 & 1.17 & 0.678 \\
\hline Level-II hospital & 1.57 & 1.42 & 1.74 & $<0.001$ \\
\hline Western Medicine hospital & 0.56 & 0.50 & 0.64 & $<0.001$ \\
\hline $\begin{array}{l}\text { Admission from } \\
\text { emergency department }\end{array}$ & 1.14 & 1.07 & 1.22 & $<0.001$ \\
\hline Diabetes & 0.94 & 0.87 & 1.01 & 0.097 \\
\hline Hypertension & 1.07 & 0.94 & 1.22 & 0.276 \\
\hline CKD stage 2 and 3 & 1.24 & 1.14 & 1.36 & $<0.001$ \\
\hline Malignancy & 2.03 & 1.86 & 2.23 & $<0.001$ \\
\hline Cirrhosis & 1.75 & $\mathrm{I} .45$ & 2.11 & $<0.001$ \\
\hline Atrial fibrillation & 1.21 & 1.12 & 1.31 & $<0.001$ \\
\hline Ischemic heart disease & 0.94 & 0.88 & 1.01 & 0.087 \\
\hline ICU admission & 0.81 & 0.76 & 0.87 & $<0.001$ \\
\hline Shock & 1.45 & 1.42 & 1.49 & $<0.001$ \\
\hline Pneumonia & 1.56 & 1.33 & 1.83 & $<0.001$ \\
\hline Intra-abdominal infection & 1.61 & $\mathrm{I} .4 \mathrm{I}$ & 1.83 & $<0.001$ \\
\hline Bloodstream infection & 1.34 & 1.12 & 1.59 & 0.001 \\
\hline CNS infection & 2.27 & 1.20 & 4.30 & 0.012 \\
\hline Respiratory insufficiency & 1.87 & 1.75 & 2.00 & $<0.001$ \\
\hline Acute liver injury & 1.01 & 0.94 & 1.09 & 0.728 \\
\hline DIC & 1.21 & 0.98 & 1.50 & 0.074 \\
\hline
\end{tabular}

In these two time periods, there were significant differences in the patients allocation between tertiary and levelII hospitals $(\mathrm{p}<0.001)$ : more patients started to attend level-II hospitals although the vast majority of patients still went to tertiary hospitals $(10.75 \%$ versus $89.20 \%$, p < 0.001). There were more S-AKI diagnosed in patients with underlying comorbidities (hypertension, CKD, cirrhosis and malignant tumors) in the post-KDIGO guideline era, and more patients were also more likely to be admitted in ICU $(p<0.001)$. Pneumonia $(p<0.001)$ and abdominal infection $(\mathrm{p}=0.002)$ appeared to be more commonly complicated by S-AKI patients in the post KDIGO era. Although the average daily cost of patients in the latest period was higher $(p<0.001)$, the hospital stays were shorter $(p<0.001)$, making no difference in the total cost related to the hospitalization $(\mathrm{p}=0.88)$.

After adjusting for other confounders, the results from the Cox proportional hazards model showed that the risk of death was significantly higher in patients hospitalized from 2005 to 2011 than those from 2012 to 2017 $(\mathrm{HR}=1.32,95 \%$ CI $(1.22-1.43), \mathrm{P}<0.001)$.

\section{AKI Patients in Both Level-II and Tertiary Hospitals (Table 6)}

The majority of S-AKI patients (89.5\%) presented and were admitted to tertiary hospitals, despite an overall increase in presentation to level-II hospitals over the observed time period. The proportion rose from $7.56 \%$ in the pre KDIGO guideline era to $10.75 \%$ in the post KDIGO guideline era $(\mathrm{P}<0.001)$. Patients over 80 years old were mostly in level-II hospitals $(p<0.001)$ whilst tertiary hospitals had younger patients $(\mathrm{p}<0.001)$. Patients were predominantly male in both levels of the hospitals.

The ICU admission rate of S-AKI patients in level-II hospitals was significantly lower than that in tertiary hospitals $(29.77 \%$ versus $34.89 \%, \mathrm{p}<0.001)$. Compared with level-II hospitals, the proportion of patients with septic shock $(\mathrm{p}=0.006)$, abdominal infection $(\mathrm{p}<0.001)$, bloodstream infection $(p<0.001)$, respiratory failure $(p<0.001)$ and MODS $(p<0.001)$ was higher in tertiary hospitals. Despite similar length of stay was seen in both hospitals $(p=0.15)$, the total cost, and average daily cost for patients being treated in tertiary hospitals were all higher $(\mathrm{p}<0.001)$.

Both the incidence $(50.8 \%$ versus $47.7 \%, \mathrm{p}<0.001)$ and the mortality rate $(62.1 \%$ versus $54.1 \%, \mathrm{p}<0.001$, 


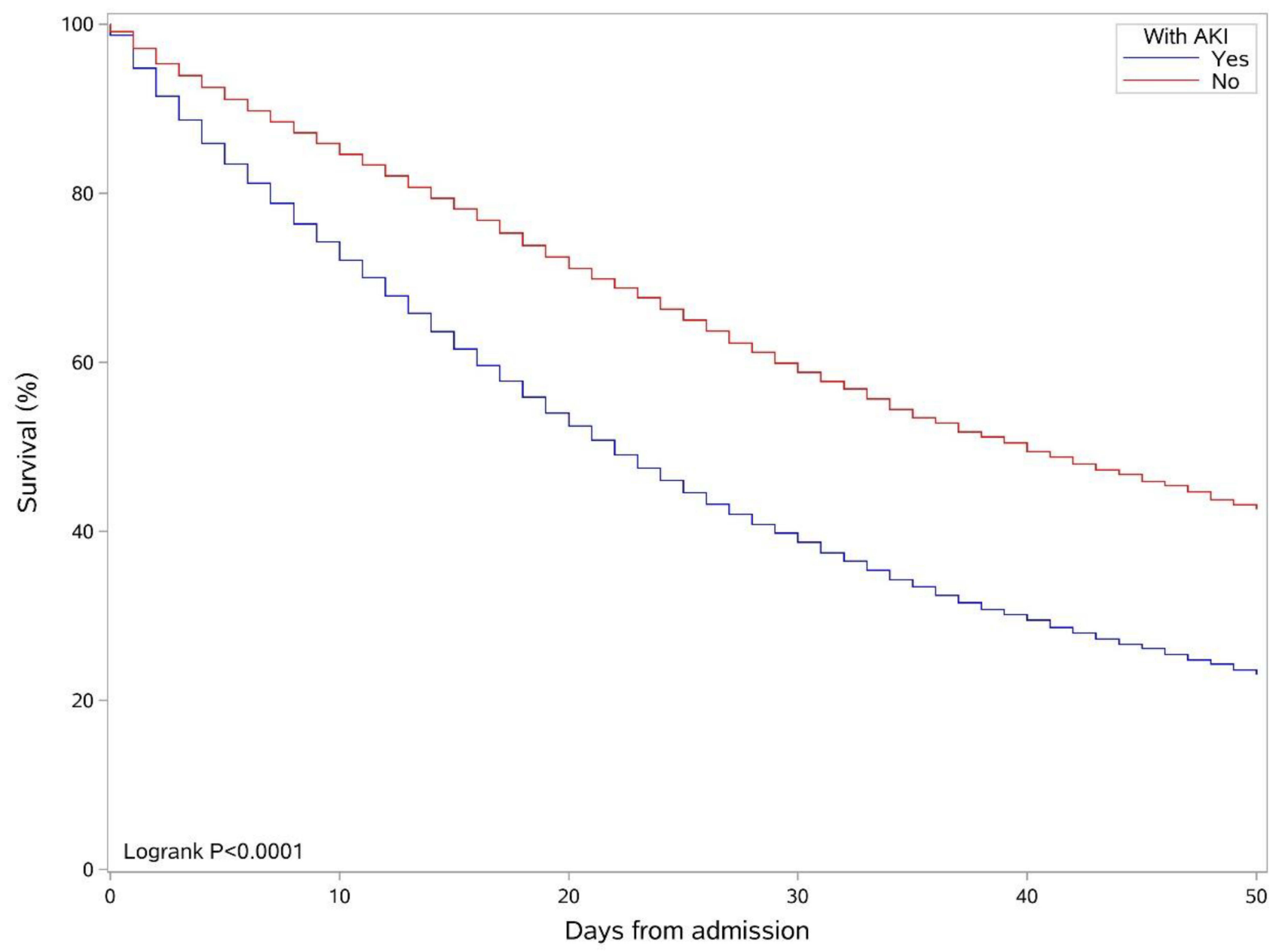

Figure I Different hospital survival probability curves of all septic patients with or without AKI.

Notes: The blue line represents the hospital survival curve of patients with AKI; the red line represents the hospital survival curve of patients without AKI.

Abbreviation: AKI, acute kidney injury.

Figure 3) of S-AKI were significantly higher in level-II hospitals than those in tertiary hospitals.

After adjusting for other confounding factors, the results from the Cox proportional hazards model showed that the risk of death was significantly higher for patients in level-II hospitals than patients in tertiary hospitals $(\mathrm{HR}=1.14,95 \%$ CI $(1.07-1.22), \mathrm{P}<0.001)$.

\section{S-AKI Patients in Traditional Chinese Medicine Hospitals and Western Medicine Hospitals (Table 7)}

The vast majority (92\%) of S-AKI patients were treated in WMHs rather than in TCMHs. The survival rate of patients treated in the WMHs was significantly higher than patients treated in TCMHs $(45.5 \%$ versus $32.5 \%, \mathrm{P}<0.001$, Figure 4). Patients from WMHs was younger than those from TCMHs $(\mathrm{P}<0.001)$.
The proportion of S-AKI patients admitted in ICU in WMHs was also higher than in TCMHs $(36.4 \%$ versus $10.1 \%, \mathrm{p}<0.001)$. The proportion of S-AKI patients with septic shock and abdominal infections in WMHs was higher than in TCMHs hospital ( $p<0.001)$. In contrast, there were less patients with lung infections in TCMHs than in WMHs $(\mathrm{P}=0.01)$. The proportion of MODS patients admitted in TCMHs was higher than in WMHs $(19.4 \%$ versus $17.1 \%$, $\mathrm{p}=0.02$ ).

The total cost, and the average daily cost were all higher in patients of WMHs $(\mathrm{p}<0.001)$. S-AKI patients from WMHs had longer length of hospitalization than patients from TCMHs $(\mathrm{P}=0.03)$.

After adjusting for other confounding factors, the results from the Cox proportional hazards model showed that the risk of death of patients in WMHs was significantly lower than that of patients in TCMHs $(\mathrm{HR}=0.70$, 95\% CI (0.65-0.75), $\mathrm{P}<0.001)$. 
Table 5 Comparison of the Epidemiology of S-AKI Between Pre and Post KDIGO Guideline for AKI Definition

\begin{tabular}{|c|c|c|c|c|}
\hline Variables & & 2005-20 I I (Year) & 20I2-2017 (Year) & $p$ value \\
\hline N (\%) & & $1468(100)$ & $|8,1| 1 \mid(100)$ & \\
\hline Death & & $798(63.9)$ & $9849(54.4)$ & $<0.001$ \\
\hline Age median [IQR] & & $75(60.5-82)$ & $78(65-85)$ & $<0.001$ \\
\hline Age stratification(years) & $\begin{array}{l}18-39 \\
40-59 \\
60-79 \\
\geq 80\end{array}$ & $\begin{array}{l}I I I(7.6) \\
238(16.2) \\
623(42.4) \\
496(33.8)\end{array}$ & $\begin{array}{l}838(4.6) \\
2419(13.4) \\
6666(36.8) \\
8188(45.2)\end{array}$ & $<0.001$ \\
\hline Gender & $\begin{array}{l}\text { Female } \\
\text { Male }\end{array}$ & $\begin{array}{l}554(37.7) \\
914(62.3)\end{array}$ & $\begin{array}{l}6741(37.2) \\
11,370(62.8)\end{array}$ & 0.69 \\
\hline Classification of insurance & $\begin{array}{l}\text { BMI } \\
\text { RCMTI } \\
\text { GMP } \\
\text { OPP } \\
\text { Others }\end{array}$ & $\begin{array}{l}750(51.1) \\
222(15.1) \\
96(6.5) \\
41(2.8) \\
359(24.5)\end{array}$ & $\begin{array}{l}10,082(55.7) \\
1260(7) \\
3215(17.8) \\
1222(6.7) \\
2332(12.9)\end{array}$ & $<0.001$ \\
\hline Hospital level & $\begin{array}{l}\text { Tertiary hospitals } \\
\text { Second-class hospital }\end{array}$ & $\begin{array}{l}1357(92.4) \\
111(7.6)\end{array}$ & $\begin{array}{l}16,163(89.2) \\
1948(10.8)\end{array}$ & $<0.001$ \\
\hline Hospital nature & $\begin{array}{l}\text { Western Medicine hospital } \\
\text { Hospital of TCM }\end{array}$ & $\begin{array}{l}1352(92.1) \\
116(7.9)\end{array}$ & $\begin{array}{l}16,656(92) \\
1455(8)\end{array}$ & 0.86 \\
\hline Comorbidities(n) & $\begin{array}{l}\text { Diabetes } \\
\text { Hypertension } \\
\text { CKD stage } 2 \text { and } 3 \\
\text { Malignancy } \\
\text { Cirrhosis }\end{array}$ & $\begin{array}{l}257(17.5) \\
422(28.7) \\
72(4.9) \\
182(12.4) \\
21(1.4)\end{array}$ & $\begin{array}{l}6763(37.3) \\
9799(54.1) \\
2872(15.9) \\
3560(19.7) \\
779(4.3)\end{array}$ & $\begin{array}{l}<0.001 \\
<0.001 \\
<0.001 \\
<0.001 \\
<0.001\end{array}$ \\
\hline ICU & & $99(6.7)$ & $6626(36.6)$ & $<0.001$ \\
\hline Types of Shock & $\begin{array}{l}\text { No shock } \\
\text { Septic shock } \\
\text { Hypovolemic shock } \\
\text { Cardiac shock } \\
\text { Two or more kinds of } \\
\text { shock }\end{array}$ & $\begin{array}{l}677(46.1) \\
72 I(49.1) \\
68(4.6) \\
4 I(2.8) \\
39(2.7)\end{array}$ & $\begin{array}{l}13,937(77) \\
2674(14.8) \\
920(5.1) \\
897(5) \\
305(1.7)\end{array}$ & $\begin{array}{l}<0.001 \\
<0.001 \\
0.45 \\
<0.001 \\
0.01\end{array}$ \\
\hline Types of infection & $\begin{array}{l}\text { Pneumonia } \\
\text { Urinary infection } \\
\text { Intra-abdominal infection } \\
\text { CRBSI } \\
\text { CNS infection } \\
\text { Others }\end{array}$ & $\begin{array}{l}842(57.4) \\
144(9.8) \\
100(6.8) \\
29(2) \\
3(0.2) \\
495(33.7)\end{array}$ & $\begin{array}{l}14,320(79.1) \\
1347(7.4) \\
1681(9.3) \\
628(3.5) \\
44(0.2) \\
2720(15)\end{array}$ & $\begin{array}{l}<0.001 \\
0.001 \\
0.002 \\
0.002 \\
0.77 \\
<0.001\end{array}$ \\
\hline $\begin{array}{l}\text { Types of organ function } \\
\text { damage }\end{array}$ & $\begin{array}{l}\text { Respiratory insufficiency } \\
\text { Acute liver injury } \\
\text { DIC } \\
\text { Metabolic encephalopathy }\end{array}$ & $\begin{array}{l}66 \mid(45) \\
131(8.9) \\
199(13.6) \\
17(1.2)\end{array}$ & $\begin{array}{l}11,123(61.4) \\
3865(21.3) \\
481(2.7) \\
221(1.2)\end{array}$ & $\begin{array}{l}<0.001 \\
<0.001 \\
<0.001 \\
0.83\end{array}$ \\
\hline
\end{tabular}


Table 5 (Continued).

\begin{tabular}{|c|c|c|c|c|}
\hline Variables & & 2005-20I I (Year) & 20I2-2017 (Year) & $p$ value \\
\hline Expenses (Yuan) & $\begin{array}{l}\text { Total expense } \\
\text { Average daily expense } \\
\text { Western medicine expense } \\
\text { Chinese medicine expense }\end{array}$ & $\begin{array}{l}62,115.33(24,502.22-134,4 \mid 9.29) \\
3859.29(2200.42-6547.85) \\
25,847.1(9208.77-58,538.56) \\
31.06(0-305.98)\end{array}$ & $\begin{array}{l}60, \mid 58.84(28,824.2|-| 2 \mid, 703.84) \\
5406.94(3280.57-87 \mid 9.52) \\
13,329.13(5430.34-28,760.24) \\
107.58(0-9 \mid 7.56)\end{array}$ & $\begin{array}{l}0.88 \\
<0.001 \\
<0.001 \\
<0.001\end{array}$ \\
\hline Length of hospital stay (day) & & $16(7-35)$ & $12(6-23)$ & $<0.001$ \\
\hline
\end{tabular}

Note: Values are expressed as the median (interquartile range and $\mathrm{N}(\%)$ ).

Abbreviations: BMI, basic medical insurance; RCMTI, rural cooperative medical treatment insurance; GMP, governmental medical payment; OPP, out-of-pocket payments; ER, emergency room; CRBSI, Catheter Related Blood Stream Infection; CNS, central nervous system; DIC, disseminated intravascular coagulation; TCM, traditional Chinese medicine.

\section{Discussion}

In this study, we analyzed the epidemiology of S-AKI in all inpatients, including ICU and non-ICU patients from 158 hospitals in Beijing, China from January 2005 to December 2017. We found the overall incidence of
S-AKI was $48.1 \%$, with $59.2 \%$ in ICU patients and $31.6 \%$ in non-ICU patients, respectively; and overall mortality rate of $55 \%$ among the S-AKI patients. Pneumonia was the most likely source of sepsis in this cohort. The reported incidence of S-AKI was higher after KDIGO

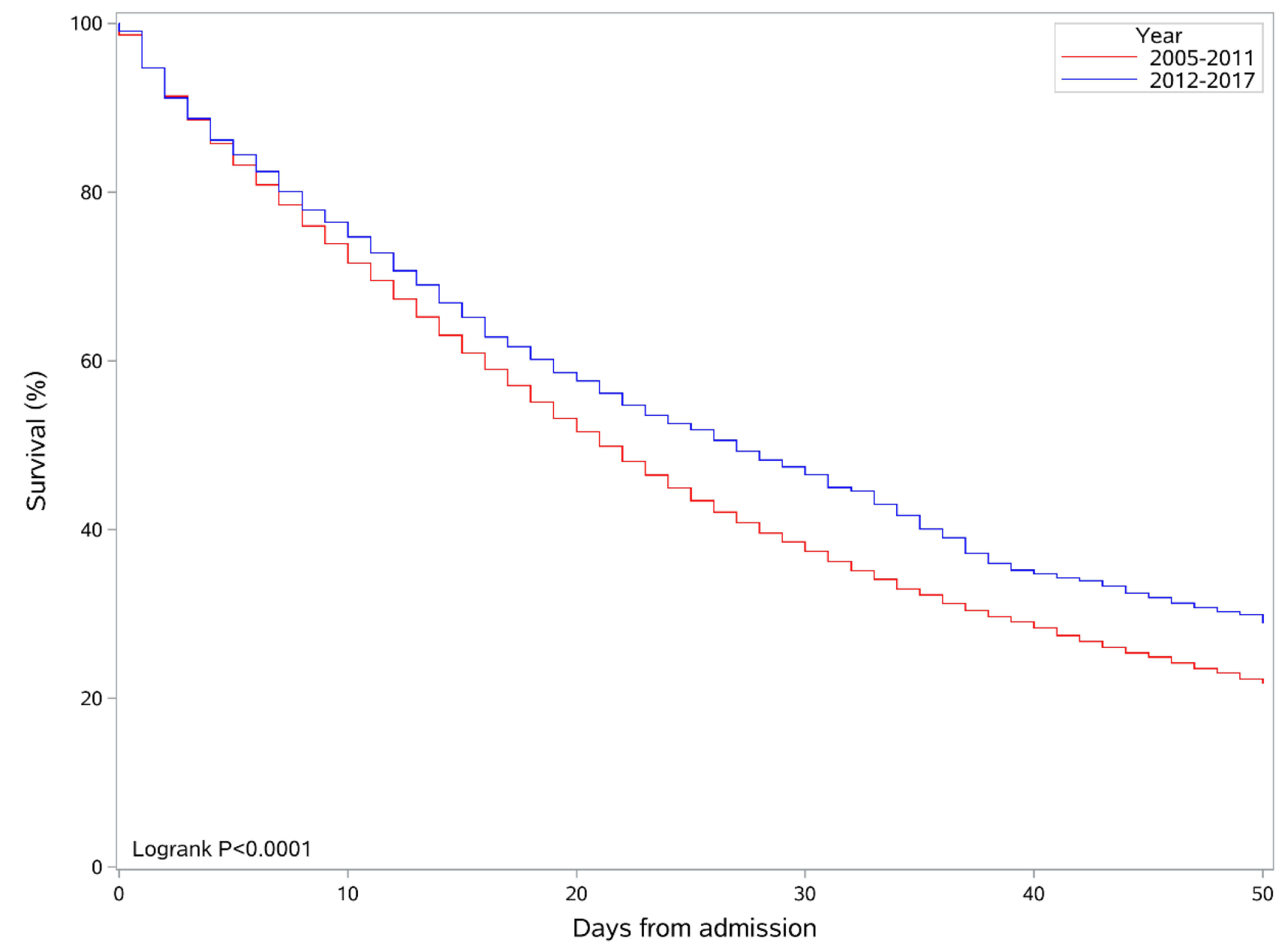

Figure 2 The hospital survival probability curves of patients with S-AKI classified according to admission date.

Notes: The blue line represents the hospital survival curve of S-AKI patients hospitalized from the year of 2005 to 201 I; the red line represents the hospital survival curve of S-AKI patients hospitalized from the year of 2012 to 2017.

Abbreviation: S-AKI, sepsis-associated acute kidney injury. 
Table 6 Comparison of the Epidemiology of S-AKI Between Level-II and Tertiary Hospitals

\begin{tabular}{|c|c|c|c|c|}
\hline Variables & & Level-II Hospital & Tertiary Hospitals & $p$ value \\
\hline N (\%) & & $2059(100)$ & $17,520(100)$ & \\
\hline Death & & $1277(62.7)$ & $9370(54.1)$ & $<0.001$ \\
\hline Age median [IQR] & & $80(70-85)$ & $78(64-84)$ & $<0.001$ \\
\hline Age stratification(years) & $\begin{array}{l}18-39 \\
40-59 \\
60-79 \\
\geq 80\end{array}$ & $\begin{array}{l}45(2.2) \\
213(10.3) \\
737(35.8) \\
1064(51.7)\end{array}$ & $\begin{array}{l}904(5.2) \\
2444(13.9) \\
6552(37.4) \\
7620(43.5)\end{array}$ & $<0.001$ \\
\hline Gender & $\begin{array}{l}\text { Female } \\
\text { Male }\end{array}$ & $\begin{array}{l}817(39.7) \\
1242(60.3)\end{array}$ & $\begin{array}{l}6478(37) \\
11,042(63)\end{array}$ & 0.02 \\
\hline Classification of insurance & $\begin{array}{l}\text { BMI } \\
\text { RCMTI } \\
\text { GMP } \\
\text { OPP } \\
\text { Others }\end{array}$ & $\begin{array}{l}1384(67.2) \\
263(12.8) \\
193(9.4) \\
96(4.7) \\
123(6)\end{array}$ & $\begin{array}{l}9448(53.9) \\
1219(7) \\
3118(17.8) \\
1167(6.7) \\
2568(14.7)\end{array}$ & $<0.001$ \\
\hline Hospital nature & $\begin{array}{l}\text { Western Medicine hospital } \\
\text { Hospital of TCM }\end{array}$ & $\begin{array}{l}1967(95.5) \\
92(4.5)\end{array}$ & $\begin{array}{l}|6,04|(91.6) \\
\mid 479(8.4)\end{array}$ & $<0.001$ \\
\hline Time Period & $\begin{array}{l}2005-2011 \\
2012-2017\end{array}$ & $\begin{array}{l}\text { III(5.4) } \\
1948(94.6)\end{array}$ & $\begin{array}{l}1357(7.7) \\
16,163(92.3)\end{array}$ & $<0.001$ \\
\hline Comorbidities(n) & $\begin{array}{l}\text { Diabetes } \\
\text { Hypertension } \\
\text { CKD stage } 2 \text { and } 3 \\
\text { Malignancy } \\
\text { Cirrhosis }\end{array}$ & $\begin{array}{l}719(34.9) \\
1085(52.7) \\
403(19.6) \\
258(12.5) \\
40(1.9)\end{array}$ & $\begin{array}{l}630 I(36) \\
9136(52 . I) \\
254 I(14.5) \\
3484(19.9) \\
760(4.3)\end{array}$ & $\begin{array}{l}0.35 \\
0.64 \\
<0.001 \\
<0.001 \\
<0.001\end{array}$ \\
\hline ICU & & $613(29.8)$ & $6112(34.9)$ & $<0.001$ \\
\hline Types of shock & $\begin{array}{l}\text { No shock } \\
\text { Septic shock } \\
\text { Hypovolemic shock } \\
\text { Cardiac shock } \\
\text { Two or more kinds of } \\
\text { shock }\end{array}$ & $\begin{array}{l}1593(77.4) \\
312(15.2) \\
108(5.2) \\
78(3.8) \\
31(1.5)\end{array}$ & $\begin{array}{l}|3,02|(74.3) \\
3083(\mid 7.6) \\
880(5) \\
860(4.9) \\
313(1.8)\end{array}$ & $\begin{array}{l}0.003 \\
0.006 \\
0.66 \\
0.02 \\
0.36\end{array}$ \\
\hline Types of infection & $\begin{array}{l}\text { Pneumonia } \\
\text { Urinary infection } \\
\text { Intra-abdominal infection } \\
\text { CRBSI } \\
\text { CNS infection } \\
\text { Others }\end{array}$ & $\begin{array}{l}1582(76.8) \\
122(5.9) \\
131(6.4) \\
22(1.1) \\
3(0.1) \\
382(18.6)\end{array}$ & $\begin{array}{l}13,580(77.5) \\
1369(7.8) \\
1650(9.4) \\
635(3.6) \\
44(0.3) \\
2833(16.2)\end{array}$ & $\begin{array}{l}0.49 \\
0.002 \\
<0.001 \\
<0.001 \\
0.36 \\
0.006\end{array}$ \\
\hline $\begin{array}{l}\text { Types of organ function } \\
\text { damage }\end{array}$ & $\begin{array}{l}\text { Respiratory insufficiency } \\
\text { Acute liver injury } \\
\text { DIC } \\
\text { Metabolic encephalopathy }\end{array}$ & $\begin{array}{l}1064(51.7) \\
286(13.9) \\
37(1.8) \\
28(1.4)\end{array}$ & $\begin{array}{l}10,720(61.2) \\
3710(21.2) \\
643(3.7) \\
210(1.2)\end{array}$ & $\begin{array}{l}<0.001 \\
<0.001 \\
<0.001 \\
0.53\end{array}$ \\
\hline
\end{tabular}


Table 6 (Continued).

\begin{tabular}{|c|c|c|c|c|}
\hline Variables & & Level-II Hospital & Tertiary Hospitals & $p$ value \\
\hline Expenses (Yuan) & $\begin{array}{l}\text { Total expense } \\
\text { Average daily expense } \\
\text { Western medicine expense } \\
\text { Chinese medicine expense }\end{array}$ & $\begin{array}{l}41,047.75(|17,598.47-92,27| .23) \\
3822.54(2297.78-6014.34) \\
9085.04(3349.65-22,224.82) \\
78.04(0-650.52)\end{array}$ & $\begin{array}{l}62,702.87(30,227.13-125,862.83) \\
5499.86(3329.52-8886.23) \\
\mid 4,510.38(5984.67-31,3 \mid 4.93) \\
98.49(0-869.4)\end{array}$ & $\begin{array}{l}<0.001 \\
<0.001 \\
<0.001 \\
0.19\end{array}$ \\
\hline Length of hospital stay (day) & & $13(4-25)$ & $13(6-23)$ & 0.15 \\
\hline
\end{tabular}

Note: Values are expressed as the median (interquartile range and $\mathrm{N}(\%)$ ).

Abbreviations: BMI, basic medical insurance; RCMTI, rural cooperative medical treatment insurance; GMP, governmental medical payment; OPP, out-of-pocket payments; ER, emergency room; CRBSI, Catheter Related Blood Stream Infection; CNS, central nervous system; DIC, disseminated intravascular coagulation; TCM, traditional Chinese medicine.

guideline being used to define AKI. The majority of AKI patients in this study was admitted to tertiary teaching hospitals and western Medicine hospitals.

There is an increasing incidence of AKI worldwide. ${ }^{1-5}$ The current literature reported incidence of S-AKI is from $39.4 \%$ to $60.7 \%$ in ICU, which is consistent with our finding $(48 \%)^{22,23}$

According to the nationwide survey regarding AKI across Mainland China, $72 \%$ of AKI episodes occurred in the non-ICU setting, which reminds us that the occurrence of AKI outside the ICU requires more attention. ${ }^{17}$

Our study showed that certain demographic characteristics, including advanced age, male and the presence of comorbidities such as hypertension, CKD and liver cirrhosis, and severity of the diseases as assessed by requirements for ICU admission and shock status were significantly associated with the incidence of AKI. These results were consistent with previous studies. ${ }^{14,24}$ However, an Australian S-AKI study revealed female patients had increased risk to develop AKI, ${ }^{2}$ whereas some other studies showed no significant difference in age or sex. ${ }^{4,13}$ These inconsistent findings may be due to different population and demographic characteristics in each study.

The overall in-hospital mortality among S-AKI in this cohort was $55 \%$, which was higher than the results from previous studies. ${ }^{4,25}$ The healthcare system in China is different from some developed countries where patients can have free access to certain procedures such as dialysis therapy, ${ }^{22}$ which may be a potential factor contributing to higher mortality rates.

Our study shows that hypertension is a risk factor for S-AKI, and $52.2 \%$ of S-AKI patients have hypertension, which are consistent with previous studies. ${ }^{26,27}$ Approximately, 244.5 million of the Chinese adult population $\geq 18$ years of age have been diagnosed with hypertension, which contributes to a significant burden of disease. $^{27}$

Hypertension is both a cause and effect of CKD and contributes to its progression. RAAS-induced injury is associated with lower glomerular filtration rate (GFR), lower renal blood flow, kidney fibrosis, up-regulation of sodium transporters, which results in both the structural and functional changes of the kidney. ${ }^{28-31}$ In the course of blood pressure intervention, some medications used can also increase the incidence of AKI. A nested case-control study showed that a triple therapy combination consisting of diuretics with angiotensin converting enzyme inhibitors or angiotensin receptor blockers and NSAIDs (Nonsteroidal anti-inflammatory drugs) was associated with an increased risk of AKI. Although antihypertensive drugs have cardiovascular benefits, vigilance may be warranted when they are used concurrently. ${ }^{32}$

Hepatitis B virus (HBV) infection is a serious problem in China, contributing to more than one-third of the world's HBV-infected people (approximately 93 million). ${ }^{33}$ Liver cirrhosis is a progressive condition in chronic hepatitis B. AKI occurs in approximately $19 \%$ of hospitalized patients with cirrhosis from previous research. ${ }^{34-36}$

Almost all the causes of AKI in patients with cirrhosis are prerenal and intrarenal, accounting for approximately $68 \%$ and $32 \%$ of the cases. ${ }^{37,38}$ Cirrhosis is characterized by reduced systemic vascular resistance due to splanchnic arterial vasodilation. ${ }^{39}$ In early stages of the disease, splanchnic vasodilation is moderate and reduced systemic vascular resistance is balanced by increased cardiac output. In advanced stages, vasodilation is more pronounced because of increased synthesis of vasodilator factors which cannot be balanced by an increase in cardiac output. ${ }^{40}$ Eventually, changes in hemodynamics in the kidney and altered autoregulation of kidney blood flow contribute to decreased glomerular filtration rate (GFR) leading to acute 


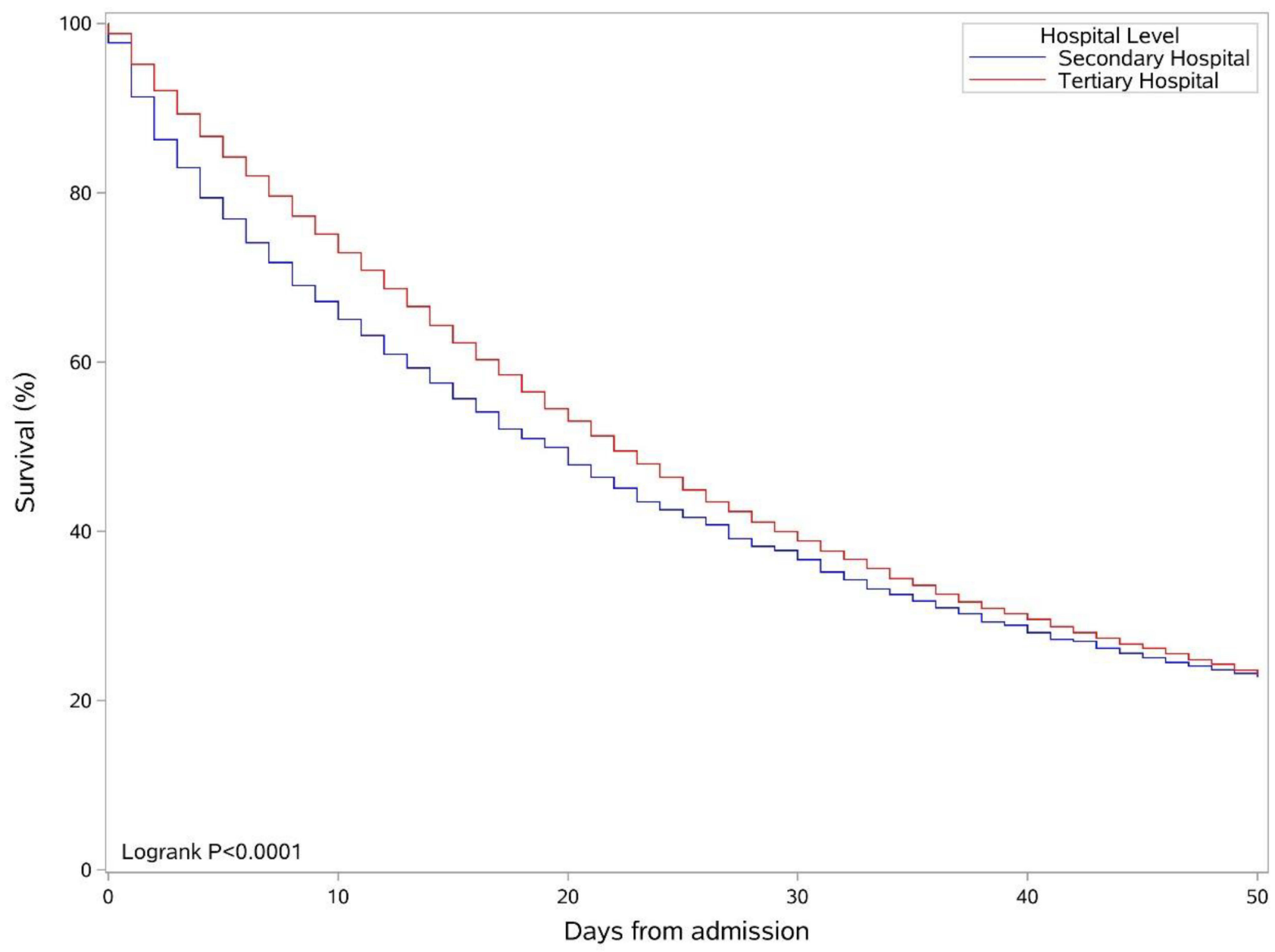

Figure 3 The hospital survival probability curves of patients with S-AKI classified according to hospital levels.

Notes: The blue line represents the hospital survival curve of S-AKI patients treated in the secondary hospital; the red line represents the hospital survival curve of S-AKI patients treated in the tertiary hospital.

Abbreviation: S-AKI, sepsis-associated acute kidney injury.

kidney injury. Moreover, these patients are often exposed to nephrotoxic agents such as nonsteroidal antiinflammatory drugs, contrast agents, and aminoglycosides, ${ }^{41}$ which are directly toxic to renal tubules, eventually leading to acute tubular necrosis. Vasoconstrictive agents (terlipressin in particular) are the first-line treatment option. Liver transplantation is the only curative treatment of AKI due to cirrhosis. ${ }^{42,43}$

\section{Comparison of the Incidence of S-AKI Between Pre and Post KDIGO Guideline for AKI Definition}

There have been multiple attempts to universally standardize the classification and definition of AKI to enable consistent diagnosis and treatment across different healthcare systems. These classifications include the RIFLE
(Risk, Injury, Failure, Loss, End Stage), AKIN (Acute Kidney Injury Network) and KDIGO (Kidney Disease: Improving Global Outcomes) which have been good predictors of mortality in critically ill patients. ${ }^{44}$ Despite slight variations in the definitions, A systematic review performed by Thomas et al has found that these definitions do not significantly differ in their predictive outcomes. ${ }^{45}$

The introduction of new AKI definition in the KDIGO guideline attempted to unify the definition of AKI of both the RIFLE and AKIN criteria. In our study, we found that the change in definitions used may have attributed to an increase in incidence of AKI. According to the AKIN criteria, the incidence of S-AKI in our cohort of patients was $39.7 \%$ from the period of 2005-2011. When the updated KDIGO criteria was used, the incidence was higher (48.9\%) from the period of 2012-2017. This 
Table 7 Comparison Between Traditional Chinese Medicine Hospitals and Western Medicine Hospitals

\begin{tabular}{|c|c|c|c|c|}
\hline Variables & & TCMHs & WMHs & $p$ value \\
\hline$N(\%)$ & & I573(8.0) & $18,006(92.0)$ & \\
\hline Survivors(n) & & $512(32.9)$ & $8200(46.1)$ & $<0.001$ \\
\hline Age median $[\mathrm{IQR}]$ & & $80(7 \mid-85)$ & $78(64-84)$ & $<0.001$ \\
\hline Age stratification(years) & $\begin{array}{l}18-39 \\
40-59 \\
60-79 \\
\geq 80\end{array}$ & $\begin{array}{l}24(1.5) \\
133(8.4) \\
590(37.5) \\
826(52.5)\end{array}$ & $\begin{array}{l}924(5.1) \\
2526(14) \\
6703(37.2) \\
7853(43.6)\end{array}$ & $<0.001$ \\
\hline Gender & $\begin{array}{l}\text { Female } \\
\text { Male }\end{array}$ & $\begin{array}{l}653(41.5) \\
920(58.5)\end{array}$ & $\begin{array}{l}6642(36.9) \\
11,364(63.1)\end{array}$ & $<0.001$ \\
\hline Classification of insurance & $\begin{array}{l}\text { BMI } \\
\text { RCMTI } \\
\text { GMP } \\
\text { OPP } \\
\text { Others }\end{array}$ & $\begin{array}{l}1166(74.2) \\
109(6.9) \\
133(8.4) \\
113(7.2) \\
52(3.3)\end{array}$ & $\begin{array}{l}9659(53.6) \\
1376(7.6) \\
3173(17.6) \\
1148(6.4) \\
2650(14.7)\end{array}$ & $<0.001$ \\
\hline Hospital level & $\begin{array}{l}\text { Tertiary hospitals } \\
\text { Second-class hospital }\end{array}$ & $\begin{array}{l}|48|(94.2) \\
92(5.8)\end{array}$ & $\begin{array}{l}16,038(89.1) \\
1968(10.9)\end{array}$ & $<0.001$ \\
\hline Time interval(year) & $\begin{array}{l}2005-2011 \\
2012-2017\end{array}$ & $\begin{array}{l}117(7.4) \\
1456(92.6)\end{array}$ & $\begin{array}{l}|35|(7.5) \\
\mid 6,655(92.5)\end{array}$ & \\
\hline Comorbidities(n) & $\begin{array}{l}\text { Diabetes } \\
\text { Hypertension } \\
\text { CKD stage } 2 \text { and } 3 \\
\text { Malignancy } \\
\text { Cirrhosis }\end{array}$ & $\begin{array}{l}521(33.1) \\
828(52.6) \\
299(19) \\
240(15.2) \\
22(1.4)\end{array}$ & $\begin{array}{l}6503(36.1) \\
9399(52.1) \\
2645(14.7) \\
3510(19.5) \\
778(4.3)\end{array}$ & $\begin{array}{l}0.02 \\
0.73 \\
<0.001 \\
<0.001 \\
<0.001\end{array}$ \\
\hline ICU & & $159(10.1)$ & $6566(36.4)$ & $<0.001$ \\
\hline Types of shock & $\begin{array}{l}\text { No shock } \\
\text { Septic shock } \\
\text { Hypovolemic shock } \\
\text { Cardiac shock } \\
\text { Two or more kinds of shock }\end{array}$ & $\begin{array}{l}1294(82.2) \\
182(11.6) \\
58(3.7) \\
60(3.8) \\
18(1.1)\end{array}$ & $\begin{array}{l}13,323(73.9) \\
3240(18) \\
930(5.2) \\
879(4.9) \\
327(1.8)\end{array}$ & $\begin{array}{l}<0.001 \\
<0.001 \\
0.01 \\
0.06 \\
0.05\end{array}$ \\
\hline Types of infection & $\begin{array}{l}\text { Pneumonia } \\
\text { Urinary infection } \\
\text { Intra-abdominal infection } \\
\text { CRBSI } \\
\text { CNS infection } \\
\text { Others }\end{array}$ & $\begin{array}{l}1260(80) \\
106(6.7) \\
86(5.5) \\
55(3.5) \\
3(0.2) \\
256(16.3)\end{array}$ & $\begin{array}{l}13,916(77.2) \\
1386(7.7) \\
1696(9.4) \\
602(3.3) \\
44(0.2) \\
2973(16.5)\end{array}$ & $\begin{array}{l}0.01 \\
0.17 \\
<0.001 \\
0.74 \\
0.68 \\
0.81\end{array}$ \\
\hline Types of organ function damage & $\begin{array}{l}\text { Respiratory insufficiency } \\
\text { Acute liver injury } \\
\text { DIC } \\
\text { Metabolic encephalopathy }\end{array}$ & $\begin{array}{l}850(54) \\
359(22.8) \\
54(3.4) \\
34(2.2)\end{array}$ & $\begin{array}{l}10,947(60.7) \\
364 \mid(20.2) \\
626(3.5) \\
204(1.1)\end{array}$ & $\begin{array}{l}<0.001 \\
0.01 \\
0.93 \\
<0.001\end{array}$ \\
\hline Expenses (Yuan) & $\begin{array}{l}\text { Total expense } \\
\text { Average daily expense } \\
\text { Western medicine expense } \\
\text { Chinese medicine expense }\end{array}$ & $\begin{array}{l}46,013.3(20,515.42-94,055.27) \\
4166.6(2721.57-6398.43) \\
9744.02(3425.73-21,786.67) \\
995.2(182.92-3489.11)\end{array}$ & $\begin{array}{l}61,667.38(29,297.85-\mid 25,052.5) \\
54|3.7|(3248.88-8781.84) \\
14,285.38(5896.72-31,166.39) \\
73.09(0-686.88)\end{array}$ & $\begin{array}{l}<0.001 \\
<0.001 \\
<0.001 \\
<0.001\end{array}$ \\
\hline Length of hospital stay (day) & & $13(5-22)$ & $13(6-23)$ & 0.03 \\
\hline
\end{tabular}

Note: Values are expressed as the median (interquartile range and $\mathrm{N}(\%)$ ).

Abbreviations: BMI, basic medical insurance; RCMTI, rural cooperative medical treatment insurance; GMP, governmental medical payment; OPP, out-of-pocket payments; ER, emergency room; CRBSI, Catheter Related Blood Stream Infection; CNS, central nervous system; DIC, disseminated intravascular coagulation; TCM, traditional Chinese medicine. 


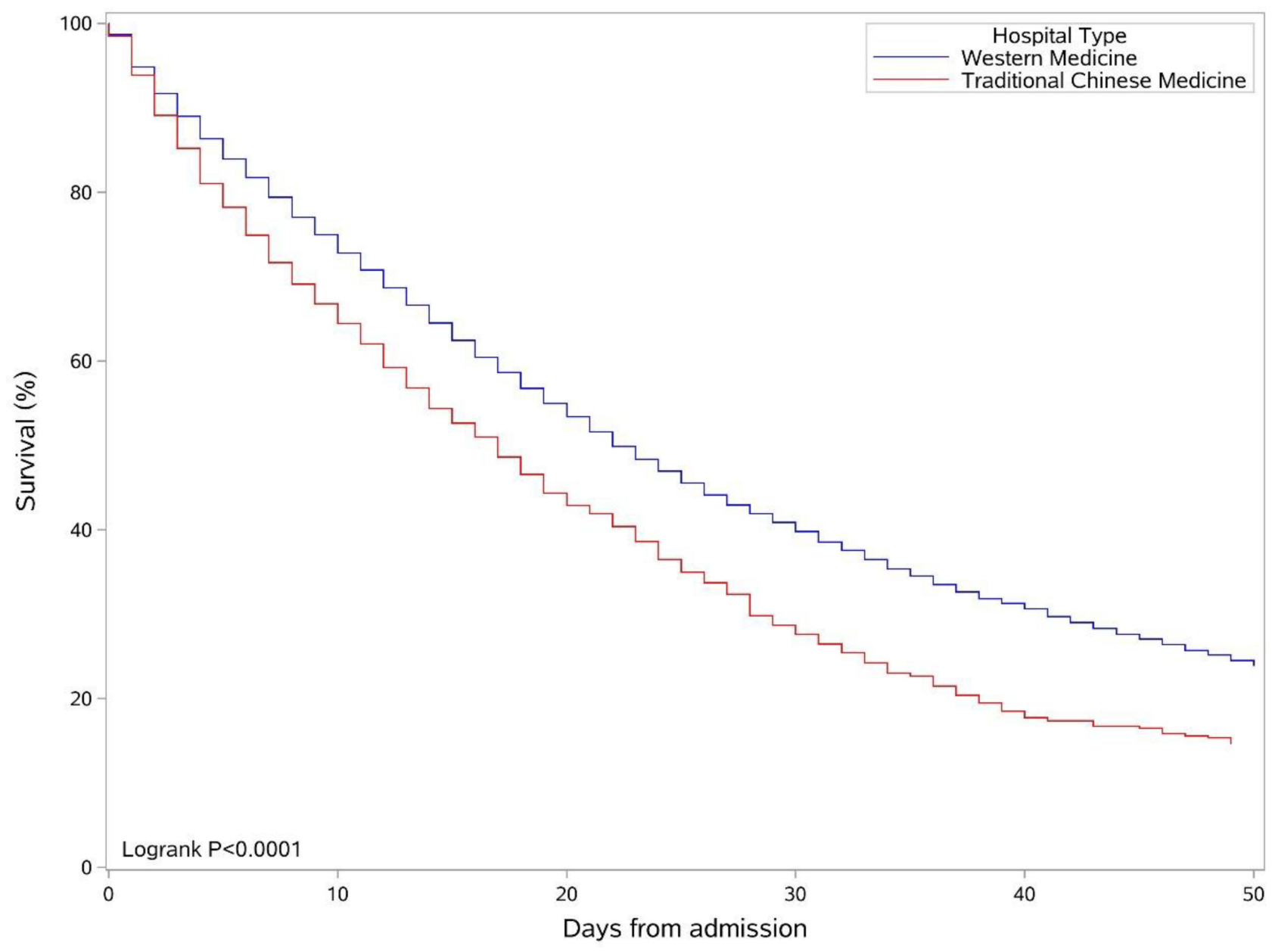

Figure 4 The hospital survival probability curves of patients with S-AKI classified according to different hospital types.

Notes: The blue line represents the hospital survival curve of S-AKI patients treated in Western Medicine hospitals; the red line represents the hospital survival curve of S-AKI patients treated in Traditional Chinese Medicine hospitals.

Abbreviation: S-AKI, sepsis-associated acute kidney injury.

finding is similar to previous studies ${ }^{46,47}$ which compared utilization of different classifications and found that the AKIN classification had consistently reported lower incidence levels when compared with KDIGO and RIFLE classifications. However, the higher incidence in our study in the later time period may also be attributed to aging population and the increased incidence of multiple chronic illnesses. Despite the increase in incidence, the mortality rates declined from pre $(63.9 \%)$ to post (54.4\%) KDIGO guideline era.

The declining mortality rates post the KDOGO guideline era may be due to multiple factors including standardized classifications of AKI leading to early recognition and treatment by clinicians; ${ }^{48-50}$ significant advances in supportive medical technologies such as continuous renal replacement therapy (CRRT) and other measures for treating sepsis, such as early appropriate antibiotic administration and fluid resuscitation, $;^{50,51}$ as well as more patients seeking treatment in tertiary hospital centers where they have access to the highest level of medical staff and equipment. ${ }^{52}$

\section{Outcomes Between Level-II and Tertiary Hospitals}

To the best of our knowledge, there are limited literature that provide direct comparisons of the mortality rates between tertiary hospitals and those with limited healthcare resources such as level-II, rural or peripheral hospitals. The incidence of S-AKI in level-II and tertiary hospitals in this study were similar, $50.8 \%$ and $47.7 \%$, respectively. The mortality rate in level-II hospitals was $9 \%$ higher than that in tertiary hospitals.

The higher mortality rates in level-II hospitals in our cohort could be attributed to two main reasons. Firstly, the 
proportion of patients over 80-year-old in level-II hospitals was higher than that in tertiary hospitals $(51.7 \%$ versus $43.5 \%$ ). Secondly, there is a limited access to interventional management strategies in level-II hospitals such as renal replacement therapy. For those who had clinical indications for being transferred to a tertiary hospital for advanced treatment but were unable to be transferred in a timely fashion (eg due to bed block or change of the treatment goal to conservative treatments), they would only be able to receive limited treatment based on the hospital resource.

\section{Traditional Chinese Medicine Hospitals (TCMHs) versus Western Medical Hospitals (WMHs)}

In China, the health care system is uniquely divided into the western and traditional Chinese medical system. ${ }^{53}$ The Chinese cultural heritage has traditionally focused on the administration of traditional Chinese service and products (such as medicinal herbs, acupuncture and dietary therapy), however, there has been a shift by the Chinese government to "integrate both Chinese and Western medicine" through the implementation of modern medicine and technologies within TCMHs since the 1970's. ${ }^{54}$

A distinct aspect of this study is the comparison of the patient outcomes between TCMHs and WMHs. In our study, the majority of the patients (92\%) were diagnosed and treated with S-AKI in WMHs. Furthermore, the number of patients admitted to WMHs was 5.6 times that of the TCMHs in 2018 alone. This can be explained by fewer availability of TCMHs when compared to WMHs. However, in both categories of hospitals, the incidence of AKI is increasing annually. The mortality rate of S-AKI patients in WMHs is lower than that of patients in TCMHs. This can be explained by more sufficient means of supporting and treating patients in WMHs. This is likely to explain higher costs for patients receiving treatment in WMHs compared to patients in TCMHs.

In addition, many Chinese patients, especially the elderly, take Chinese herbal medicine as the first choice for treating diseases. However, some herbal medicines are also known to cause nephrotoxicity, which is often overlooked by the treating physicians and patients. ${ }^{55}$ The incidence of acute kidney injury induced by Chinese herbal medicines is difficult to assess. One cross-sectional survey of AKI in China found that Chinese patients (71.6\%) were more likely to be exposed to traditional Chinese medicine before and during AKI than patients from developed countries $(20-50 \%){ }^{16}$ This high proportion of exposure to nephrotoxic agents is probably consistent with the increasing incidence of drug-induced disease in China. ${ }^{56}$ Therefore, the use of Chinese herbal medicine may be a double-edged sword in the treatment of AKI patients.

\section{Strengths and Limitations}

This large retrospective cohort study assessed epidemiological features of S-AKI at the municipal level, in China, consisting 40,720 patients and 158 hospitals. To our knowledge, this is the first study investigated the impact of levels (level-II versus tertiary teaching hospitals) and types of hospitals (TCMHs versus western medicine hospitals) as well as definition of AKI on incidence, and risk factors of S-AKI.

Our study has several limitations. Firstly, our study used a large administrative dataset with insufficient information in the stages of AKI, use of renal replacement therapy (RRT) and the long-term prognosis of renal function, which may impact on morbidity and hospital mortality. Secondly, even though a number of statistical analyses were conducted in our study, potential confounders still exist, which may have an impact on the study finding. Thirdly, due to the nature of the study using an administrative dataset, a diagnosis of S-AKI in this study was made when based upon a septic patient developed an episode of AKI at the same time. This may overestimate the incidence of S-AKI. Fourthly, the use of different definitions of AKI during our study can lead to variations in overall incidence. From an epidemiologic standpoint it may be difficult to compare results of future studies which highlights the necessity for a standardized and comprehensive AKI classification system. Finally, there were large differences in the patients' numbers in different time periods and different levels and categories of hospitals, so the results of the comparisons may have a certain deviation.

\section{Conclusions}

AKI is a common complication in all hospitalized patients with sepsis, and its incidence increases over time, especially when ICU admission is required. Our findings provide valuable information about the epidemiology of hospitalized patients with S-AKI in Beijing, China. Exploring interventional strategies to address modifiable risk factors will be important to reduce incidence and mortality of S-AKI. 


\section{Abbreviations}

AKI, Acute kidney injury; S-AKI, Sepsis-associated acute kidney injury; ICU, Intensive care unit; ESKD, End-stage kidney disease; TCMHs, Traditional Chinese medicine hospitals; WMHs, Western medicine hospitals; CKD, Chronic kidney disease; AKIN, Acute Kidney Injury Network; KDIGO, Kidney Disease Improving Global Outcomes; RIFLE, Risk, Injury, Failure, Loss, End Stage; CRRT, Continuous renal replacement therapy; DM, Diabetes mellitus; HT, Hypertension.

\section{Data Sharing Statement}

The datasets used and/or analyzed during the current study are available on reasonable request from the corresponding author, Dr. Meili Duan (Email: dmeili@ccmu.edu.cn).

\section{Ethics Approval and Consent to Participate}

The study was approved by the research ethics committee of Beijing Friendship Hospital (Reference Number: 2020P2-089-01).

\section{Acknowledgments}

We would like to express our gratitude to Jiapeng $\mathrm{Lu}$ and Lei Dong for their aid in data cleansing. Dr Amanda Y Wang is supported by RACP Jacquot Research Establishment Fellowship, Australia.

\section{Author Contributions}

All authors made a significant contribution to the work reported, whether that is in the conception, study design, execution, acquisition of data, analysis and interpretation, or in all these areas; took part in drafting, revising or critically reviewing the article; gave final approval of the version to be published; have agreed on the journal to which the article has been submitted; and agree to be accountable for all aspects of the work.

\section{Disclosure}

Dr Amanda Ying Wang is supported by RACP Jacquot Research Establishment Fellowship, Australia. The authors report no other conflicts of interest in this work.

\section{References}

1. Nisula S, Kaukonen K-M, Vaara ST, et al. Incidence, risk factors and 90-day mortality of patients with acute kidney injury in Finnish intensive care units: the FINNAKI study. Intensive Care Med. 2013;39:420-428. doi:10.1007/s00134-012-2796-5

2. Bagshaw SM, George C, Dinu I, Bellomo R. A multi-centre evaluation of the RIFLE criteria for early acute kidney injury in critically ill patients. Nephrol Dial Transplant. 2008;23:1203-1210. doi:10.1093/ $\mathrm{ndt} / \mathrm{gfm} 744$

3. Ostermann M, Chang RW. Acute kidney injury in the intensive care unit according to RIFLE. Crit Care Med. 2007;35:1837-1843; quiz 1852. doi:10.1097/01.CCM.0000277041.13090.0A

4. Hoste EA, Bagshaw SM, Bellomo R, et al. Epidemiology of acute kidney injury in critically ill patients: the multinational AKI-EPI study. Intensive Care Med. 2015;41:1411-1423. doi:10.1007/ s00134-015-3934-7

5. Xu X, Nie S, Liu Z, et al. Epidemiology and clinical correlates of AKI in Chinese hospitalized adults. Clin J Am Soc Nephrol. 2015;10:1510-1518. doi:10.2215/CJN.02140215

6. Uchino S, Kellum JA, Bellomo R, et al. Acute renal failure in critically ill patients: a multinational, multicenter study. JAMA. 2005;294:813-818. doi:10.1001/jama.294.7.813

7. Case J, Khan S, Khalid R, Khan A. Epidemiology of acute kidney injury in the intensive care unit. Crit Care Res Pract. 2013;2013:479730.

8. Gammelager H, Christiansen CF, Johansen MB, Tønnesen E, Jespersen B, Sørensen HT. Five-year risk of end-stage renal disease among intensive care patients surviving dialysis-requiring acute kidney injury: a nationwide cohort study. Crit Care. 2013;17:R145. doi:10.1186/cc12824

9. Bellomo R, Kellum JA, Ronco C, et al. Acute kidney injury in sepsis. Intensive Care Med. 2017;43:816-828. doi:10.1007/s00134-0174755-7

10. Bagshaw SM, George C, Bellomo R; ANZICS Database Management Committee. Early acute kidney injury and sepsis: a multicentre evaluation. Crit Care. 2008;12:R47. doi:10.1186/cc6863

11. Jiang L, Zhu Y, Luo X, et al. Epidemiology of acute kidney injury in intensive care units in Beijing: the multi-center BAKIT study. BMC Nephrol. 2019;20:468. doi:10.1186/s12882-019-1660-z

12. Fan H, Zhao Y, Chen GD, Sun M, Zhu JH. Health insurance status and risk factors of mortality in patients with septic acute kidney injury in Ningbo, China. J Int Med Res. 2019;47:370-376. doi: $10.1177 / 0300060518802526$

13. Li M, Su JT, Wu SS, Wei ZH, Kong YY, Jia JD. Correlation among age, sex, and liver diseases-related mortality risk in patients with hepatitis B virus-related liver cirrhosis. Zhonghua Gan Zang Bing Za Zhi. 2021;29:403-408.

14. Kidney Disease: Improving Global Outcomes (KDIGO) Acute Kidney Injury Work Group. KDIGO clinical practice guideline for acute kidney injury. Kidney Int. 20122;(Suppl 2):1-138.

15. Bellomo R, Ronco C, Kellum JA, Mehta RL, Palevsky P; Acute Dialysis Quality Initiative workgroup. Acute renal failure-definition, outcome measures, animal models, fluid therapy and information technology needs: the Second International Consensus Conference of the Acute Dialysis Quality Initiative (ADQI) group. Crit Care. 2004;8:R204-R212. doi:10.1186/cc2872

16. Mehta RL, Kellum JA, Shah SV, et al. Acute Kidney Injury Network: report of an initiative to improve outcomes in acute kidney injury. Crit Care. 2007;11:R31. doi:10.1186/cc5713

17. Yang L, Xing G, Wang L, et al. Acute kidney injury in China: a cross-sectional survey. Lancet. 2015;386:1465-1471. doi:10.1016/ S0140-6736(15)00344-X 
18. Ali T, Khan I, Simpson W, et al. Incidence and outcomes in acute kidney injury: a comprehensive population-based study. J Am Soc Nephrol. 2007;18:1292-1298. doi:10.1681/ASN.2006070756

19. Cruz DN, Ronco C. Acute kidney injury in the intensive care unit: current trends in incidence and outcome. Crit Care. 2007;11:149. doi: $10.1186 / \mathrm{cc} 5965$

20. Lafrance JP, Djurdjev O, Levin A. Incidence and outcomes of acute kidney injury in a referred chronic kidney disease cohort. Nephrol Dial Transplant. 2010;25:2203-2209. doi:10.1093/ndt/gfq011

21. James MT, Hemmelgarn BR, Wiebe N, et al. Glomerular filtration rate, proteinuria, and the incidence and consequences of acute kidney injury: a cohort study. Lancet. 2010;376:2096-2103. doi:10.1016/ S0140-6736(10)61271-8

22. Peters E, Antonelli M, Wittebole X, et al. A worldwide multicentre evaluation of the influence of deterioration or improvement of acute kidney injury on clinical outcome in critically ill patients with and without sepsis at ICU admission: results from The Intensive Care Over Nations audit. Crit Care. 2018;22:188. doi:10.1186/s13054018-2112-z

23. Zhao Y, Yang L. Perspectives on acute kidney injury strategy in China. Nephrology (Carlton). 2018;23(Suppl 4):100-103. doi:10.1111/ nep. 13458

24. Khosla N, Soroko SB, Chertow GM, et al. Preexisting chronic kidney disease: a potential for improved outcomes from acute kidney injury. Clin J Am Soc Nephrol. 2009;4:1914-1919. doi:10.2215/CJN.01690309

25. Fiorentino M, Tohme FA, Wang S, Murugan R, Angus DC, Kellum JA. Long-term survival in patients with septic acute kidney injury is strongly influenced by renal recovery. PLoS One. 2018;13: e0198269. doi:10.1371/journal.pone.0198269

26. Selmi Y, Ariba YB, Labidi J. Epidemiology, diagnosis, and etiology of acute kidney injury in the elderly: a retrospective analysis. Saudi J Kidney Dis Transpl. 2019;30:678-685. doi:10.4103/1319-2442.261344

27. Wang Z, Chen Z, Zhang L, et al.; China Hypertension Survey Investigators. Status of hypertension in China: results from the china hypertension survey, 2012--2015. Circulation. 2018;137:2344-2356. doi:10.1161/CIRCULATIONAHA.117.032380

28. Bidani AK, Griffin KA. Pathophysiology of hypertensive renal damage: implications for therapy. Hypertension. 2004;44:595-601. doi:10.1161/01.HYP.0000145180.38707.84

29. Brantsma AH, Bakker SJ, de Zeeuw D, de Jong PE, Gansevoort RT. Urinary albumin excretion as a predictor of the development of hypertension in the general population. $J$ Am Soc Nephrol. 2005;17:331-335. doi:10.1681/ASN.2005111153

30. Kestenbaum B, Rudser KD, de Boer IH, et al. Differences in kidney function and incident hypertension: the multi-ethnic study of atherosclerosis. Ann Intern Med. 2008;148:501-508. doi:10.7326/ 0003-4819-148-7-200804010-00006

31. Almeida LF, Tofteng SS, Madsen K, Jensen BL. Role of the renin-angiotensin system in kidney development and programming of adult blood pressure. Clin Sci (Lond). 2020;134:641-656. doi:10.1042/CS20190765

32. Lapi F, Azoulay L, Yin H, Nessim SJ, Suissa S. Concurrent use of diuretics, angiotensin converting enzyme inhibitors, and angiotensin receptor blockers with non-steroidal anti-inflammatory drugs and risk of acute kidney injury: nested case-control study. BMJ. 2013;346:8525. doi:10.1136/bmj.e8525

33. Cui Y, Jia J. Update on epidemiology of hepatitis B and C in China. J Gastroenterol Hepatol. 2013;28:7-10. doi:10.1111/jgh.12220

34. Hampel H, Bynum GD, Zamora E, El-Serag HB. Risk factors for the development of renal dysfunction in hospitalized patients with cirrhosis. Am J Gastroenterol. 2001;96:2206-2210. doi:10.1111/ j.1572-0241.2001.03958.x
35. Peron JM, Bureau C, Gonzalez L, et al. Treatment of hepatorenal syndrome as defined by the international ascites club by albumin and furosemide infusion according to the central venous pressure: a prospective pilot study. Am J Gastroenterol. 2005;100:2702-2707. doi:10.1111/j.1572-0241.2005.00271.x

36. Wu CC, Yeung LK, Tsai WS, et al. Incidence and factors predictive of acute renal failure in patients with advanced liver cirrhosis. Clin Nephrol. 2006;65:28-33. doi:10.5414/CNP65028

37. Moreau R, Durand F, Poynard T, et al. Terlipressin in patients with cirrhosis and type I hepatorenal syndrome: a retrospective multicenter study. Gastroenterology. 2002;122:923-930. doi:10.1053/gast.2002.32 364

38. Fang JT, Tsai MH, Tian YC, et al. Outcome predictors and new score of critically ill cirrhotic patients with acute renal failure. Nephrol Dial Transplant. 2008;23:1961-1969. doi:10.1093/ndt/gfm914

39. Schrier RW, Arroyo V, Bernardi M, Epstein M, Henriksen JH, Rodés J. Peripheral arterial vasodilation hypothesis: a proposal for the initiation of renal sodium and water retention in cirrhosis. Hepatology. 1988;8:1151-1157. doi:10.1002/hep.1840080532

40. Ruiz-del-Arbol L, Monescillo A, Arocena C, et al. Circulatory function and hepatorenal syndrome in cirrhosis. Hepatology. 2005;42:439-447. doi:10.1002/hep.20766

41. Garcia-Tsao G. Further evidence against the use of aminoglycosides in cirrhotic patients. Gastroenterology. 1998;114:612-613. doi:10.10 16/S0016-5085(98)70551-7

42. Boyer TD, Sanyal AJ, Wong F, et al.; Investigators RS. Terlipressin plus albumin is more effective than albumin alone in improving renal function in patients with cirrhosis and hepatorenal syndrome type 1 . Gastroenterology. 2016;150:1579-1589. doi:10.1053/j.gastro.2016.02. 026

43. Cavallin M, Piano S, Romano A, et al. Terlipressin given by continuous intravenous infusion versus intravenous boluses in the treatment of hepatorenal syndrome: a randomized controlled study. Hepatology. 2016;63:983-992. doi:10.1002/hep.28396

44. Levi TM, Souza SPD, Magalhães JGD, et al. Comparison of the RIFLE, AKIN and KDIGO criteria to predict mortality in critically ill patients. Rev Bras Ter Intensiva. 2013;25:290-296. doi:10.5935/ 0103-507X.20130050

45. Thomas ME, Blaine C, Dawnay A, et al. The definition of acute kidney injury and its use in practice. Kidney Int. 2015;87:62-73. doi:10.1038/ki.2014.328

46. Sutherland SM, Byrnes JJ, Kothari M, et al. AKI in hospitalized children: comparing the pRIFLE, AKIN, and KDIGO definitions. Clin J Am Soc Nephrol. 2015;10:554-561. doi:10.2215/CJN.01900214

47. Fujii T, Uchino S, Takinami M, Bellomo R. Validation of the Kidney Disease Improving Global Outcomes criteria for AKI and comparison of three criteria in hospitalized patients. Clin J Am Soc Nephrol. 2014;9:848-854. doi:10.2215/CJN.09530913

48. Srisawat N, Murugan R, Lee M, et al. Plasma neutrophil gelatinase-associated lipocalin predicts recovery from acute kidney injury following community-acquired pneumonia. Kidney Int. 2011;80:545-552. doi:10.1038/ki.2011.160

49. Bagshaw SM, Lapinsky S, Dial S, et al. Acute kidney injury in septic shock: clinical outcomes and impact of duration of hypotension prior to initiation of antimicrobial therapy. Intensive Care Med. 2009;35:871-881. doi:10.1007/s00134-008-1367-2

50. Meersch M, Küllmar M, Schmidt C, et al. Long-term clinical outcomes after early initiation of RRT in critically ill patients with AKI. $J$ Am Soc Nephrol. 2018;29:1011-1019. doi:10.1681/ASN.2017060694

51. Zarbock A, Kellum JA, Schmidt C, et al. Effect of early vs delayed initiation of renal replacement therapy on mortality in critically ill patients with acute kidney injury: the ELAIN randomized clinical trial. JAMA. 2016;315:2190-2199. doi:10.1001/jama.2016.5828 
52. Wang ST, Lin CH, Wang JN, Wang CJ, Chen TJ, Yeh TF. A study of the referral patterns of obstetric clinics and the performance of receiving neonatal intensive care units in Taiwan. Public Health. 1997;111:149-152. doi:10.1016/S0033-3506(97)00573-8

53. Zhu D, Shi X, Nicholas S, He P, Fang X. Regional disparities in health care resources in traditional Chinese medicine county hospitals in China. PLoS One. 2020;15:e0227956. doi:10.1371/journal.pone. 0227956

54. Wang L, Suo S, Li J, et al. An investigation into traditional Chinese medicine hospitals in China: development trend and medical service innovation. Int J Health Policy Manag. 2017;6:19-25. doi:10.15171/ ijhpm.2016.72
55. Yang B, Xie Y, Guo M, Rosner MH, Yang H, Ronco C. Nephrotoxicity and Chinese herbal medicine. Clin J Am Soc Nephrol. 2018;13:1605-1611. doi:10.2215/CJN.11571017

56. Katsoulieris E, Mabley JG, Samai M, Sharpe MA, Green IC, Chatterjee PK. Lipotoxicity in renal proximal tubular cells: relationship between endoplasmic reticulum stress and oxidative stress pathways. Free Radic Biol Med. 2010;48:1654-1662. doi:10.1016/j. freeradbiomed.2010.03.021

\section{Publish your work in this journal}

The International Journal of General Medicine is an international, peer-reviewed open-access journal that focuses on general and internal medicine, pathogenesis, epidemiology, diagnosis, monitoring and treatment protocols. The journal is characterized by the rapid reporting of reviews, original research and clinical studies across all disease areas. The manuscript management system is completely online and includes a very quick and fair peer-review system, which is all easy to use. Visit http://www.dovepress.com/ testimonials.php to read real quotes from published authors. 